Algebraic $8 \mathcal{G}$ Geometric $\mathcal{T}_{\text {opology }}$

Volume 4 (2004) 473-520

Published: 8 July 2004

ATG

\title{
Combinatorial Miller-Morita-Mumford classes and Witten cycles
}

\author{
KiYoshi IGUsA
}

\begin{abstract}
We obtain a combinatorial formula for the Miller-Morita-Mumford classes for the mapping class group of punctured surfaces and prove Witten's conjecture that they are proportional to the dual to the Witten cycles. The proportionality constant is shown to be exactly as conjectured by Arbarello and Cornalba [1]. We also verify their conjectured formula for the leading coefficient of the polynomial expressing the Kontsevich cycles in terms of the Miller-Morita-Mumford classes.
\end{abstract}

AMS Classification 57N05; 55R40, 57M15

Keywords Mapping class group, fat graphs, ribbon graphs, tautological classes, Miller-Morita-Mumford classes, Witten conjecture, Stasheff associahedra

\section{Introduction}

The Miller-Morita-Mumford classes were defined by David Mumford [16] as even dimensional cohomology classes on the Deligne-Mumford compactification of the moduli space of Riemann surfaces of a fixed genus $g$. These are also referred to as tautological classes.

Around the same time Shigeyuki Morita [13] defined characteristic classes for oriented surface bundles. These are topologically defined integer cohomology classes for the mapping class group $M_{g}$, ie, the group of isotopy classes of orientation preserving diffeomorphisms of a fixed Riemann surface $\Sigma_{g}$ of genus $g$ :

$$
\kappa_{k} \in H^{2 k}\left(M_{g} ; \mathbb{Z}\right)
$$

The mapping class group has rational cohomology isomorphic to that of the (uncompactified) moduli space. Ed Miller [1] showed that Mumford's tautological classes correspond under this isomorphism to these topologically defined classes. Using work of John Harer [3] Miller showed that these cohomology 
classes $\kappa_{k}$ are algebraically independent in the stable range. This result was also obtained independently by Morita [14].

In this paper we take the topological viewpoint. We note that the topological and algebraic geometric definitions of the Miller-Morita-Mumford classes agree up to a sign of $(-1)^{k+1}$. (See [15].)

The mapping class group $M_{g}^{s}$ of genus $g$ surfaces with $s \geq 1$ boundary components (which we are allowed to rotate and permute) is classified by a space of fat graphs (also called ribbon graphs). These are defined to be finite graphs where all vertices have valence 3 or more together with a cyclic ordering of the half edges incident to each vertex. E. Witten conjectured that the Miller-MoritaMumford classes were dual to certain $2 k$-cycles in the space of fat graphs. We call these the Witten cycles and denote them by $W_{k}$. (See 3.4.) M. Kontsevich in 10] constructed other cycles in the space of fat graphs and conjectured that they could be expressed in terms of the Miller-Morita-Mumford classes.

Robert Penner [18] verified Witten's conjecture when $k=1$. However his calculation was off by a factor of 2 . The correct statement for $k=1$ was given by E. Arbarello and M. Cornalba [1]:

$$
\widetilde{\kappa}_{1}=\frac{1}{12}\left[W_{1}\right]^{*}
$$

where $\widetilde{\kappa}_{1} \in H^{2}\left(M_{g}^{s} ; \mathbb{Z}\right)$ is the adjusted Miller-Morita-Mumford class defined in 4.4

In this paper we prove the Witten conjecture for all $k \geq 0$ :

Theorem 0.1 The adjusted Miller-Morita-Mumford class $\widetilde{\kappa}_{k}$ is related to the duals $\left[W_{k}\right]^{*}$ of the Witten cycles as elements of $H^{2 k}\left(M_{g}^{s} ; \mathbb{Q}\right)$ for all $k \geq 0$ by

$$
\widetilde{\kappa}_{k}=(-1)^{k+1} \frac{(k+1) !}{(2 k+2) !}\left[W_{k}\right]^{*}
$$

as conjectured in [1].

To prove this we construct an elementary combinatorial cocycle representing the class $\widetilde{\kappa}_{k}$ (Theorem 3.13 ) and evaluate it on the cocycle $\left[W_{k}\right]$ (Theorem 5.1).

As an easy consequence of the above theorem (combining Theorem [5.1 with Corollary 3.15) we obtain the following. 
Corollary 0.2 The Kontsevich cycles $W_{k_{1}^{n_{1}} \ldots k_{r}^{n_{r}}}$ [10] are dual to polynomials in the adjusted Miller-Morita-Mumford classes with leading terms as conjectured in [1]:

$$
\left[W_{k_{1}^{n_{1}} \ldots k_{r}^{n_{r}}}\right]^{*}=\prod_{i=1}^{r} \frac{1}{n_{i} !}\left(2 \frac{\left(2 k_{i}+1\right) !}{(-1)^{k_{i}+1} k_{i} !}\right)^{n_{i}}\left(\widetilde{\kappa}_{k_{1}}\right)^{n_{1}} \cdots\left(\widetilde{\kappa}_{k_{r}}\right)^{n_{r}}+\text { lower terms. }
$$

Outline of the paper: In the first two sections we construct a $2 k$ cocycle $c_{\mathcal{Z}}^{k}$ on the category of cyclically ordered set $\mathcal{Z}$ and show that it represents the $k^{\text {th }}$ power of the Euler class on $|\mathcal{Z}| \simeq \mathbb{C} P^{\infty}$. In Section 3 we use this to define a $2 k$ cocycle $c_{\mathcal{F} a t}^{k}$ on the category of fat graphs $\mathcal{F}$ at and show that it is proportional to the dual $\left[W_{k}\right]^{*}$ of the Witten cycle $\left[W_{k}\right]$. The proportionality constant is computed at the end of this section using Stasheff associahedra. Section 4 uses Morse theory on surfaces associated to fat graphs to show that $\left[W_{k}\right]^{*}$ is proportional to the adjusted Miller-Morita-Mumford class $\widetilde{\kappa}_{k}$ on $|\mathcal{F} a t| \simeq$ $\coprod B M_{g}^{s}$. The final section computes the proportionality constant between $\widetilde{\kappa}_{k}$ and $c_{\mathcal{F} a t}^{k}$ giving the main theorems as stated above.

I would like to thank Robert Penner for explaining the Witten conjecture to me in detail many years ago. More recently I owe thanks to Jack Morava and Dieter Kotschick whose questions and comments lead to this current successful attempt at this conjecture. I am very grateful to Daniel Ruberman and Harry Tamvakis for their help in developing the original ideas for the combinatorial MillerMorita-Mumford classes. Finally, I would like to thank Nariya Kawazumi, Shigeyuki Morita and Kenji Fukaya and everyone in Tokyo and Kyoto who helped and encouraged me during the time that I was improving the results of this paper.

The main theorem of this paper has corollaries which are explained in two other papers, the third jointly with Michael Kleber. These subsequent papers $(4],[8])$ also give formulas for the "lower terms" in the above expression. At the same time a paper by Mondello [12] has appeared proving the same thing. This paper was written under NSF Grant DMS-0204386 and revised under DMS-0309480.

\section{Cyclically ordered sets}

In this section we consider the category $\mathcal{Z}$ of cyclically ordered sets and cyclically ordered monomorphisms. This category is one of a number of well-known 
models for $\mathbb{C} P^{\infty}$ and therefore its integer cohomology is a polynomial algebra in its Euler class

$$
e_{\mathcal{Z}} \in H^{2}(|\mathcal{Z}| ; \mathbb{Z})
$$

We give an explicit rational cocycle $c_{\mathcal{Z}}^{k}$ for the $k^{\text {th }}$ power $e_{\mathcal{Z}}^{k}$ of this Euler class. The crucial point is that, in order for $c_{\mathcal{Z}}^{k}$ to be non-zero on a $2 k$-simplex

$$
C_{0} \longmapsto C_{1} \longmapsto \cdots \longmapsto C_{2 k},
$$

the cyclic sets $C_{i}$ must strictly increase in size:

$$
\left|C_{0}\right|<\left|C_{1}\right|<\cdots<\left|C_{2 k}\right| .
$$

\subsection{The category $\mathcal{Z}$ of cyclically ordered sets}

By a cyclically ordered set we mean a finite non-empty set $C$ with, say, $n$ elements, together with a cyclic permutation $\sigma$ of $C$ of order $n$. Thus $C$ has $(n-1)$ ! cyclic orderings. We also use square brackets to denote cyclically ordered sets:

$$
(C, \sigma)=\left[x, \sigma(x), \sigma^{2}(x), \cdots, \sigma^{n-1}(x)\right] .
$$

To avoid set theoretic problems we assume that $C$ is a subset of some fixed infinite set.

To each cyclically ordered set $(C, \sigma)$ we can associate an oriented graph $S^{1}(C, \sigma)$ with one vertex for each element of $C$ and one directed edge $x \rightarrow y$ if $y=\sigma(x)$. Then $S^{1}(C, \sigma)$ is homeomorphic to a circle.

Any monomorphism of cyclically ordered sets $f:(C, \sigma) \rightarrow(D, \tau)$ has a degree given by

$$
\operatorname{deg}(f)=\frac{1}{|D|} \sum_{x \in C} k(x)
$$

where $k=k(x)$ is the smallest positive integer so that $f(\sigma(x))=\tau^{k}(f(x))$. The degree of $f$ is also the degree of the induced mapping $f_{*}: S^{1}(C, \sigma) \rightarrow$ $S^{1}(D, \tau)$. However, note that this is not a functor $\left((f g)_{*} \neq f_{*} g_{*}\right.$ and $\operatorname{deg}(f g) \neq$ $\operatorname{deg}(f) \operatorname{deg}(g)$ in general). For example, the degree of any monomorphism $f$ is equal to 1 if the domain has $\leq 2$ elements.

Let $\mathcal{Z}$ be the category of cyclically ordered sets $(S, \sigma)$ with morphisms $f:(S, \sigma)$ $\rightarrow(T, \tau)$ defined to be set monomorphisms $f: S \rightarrow T$ of degree 1 . 


\subsection{Linearly ordered sets}

Let $\mathcal{L}$ denote the category of finite, non-empty, linearly ordered sets and order preserving monomorphisms. Then we have a functor $J: \mathcal{L} \rightarrow \mathcal{Z}$ sending $C=$ $\left(x_{1}<\cdots<x_{n}\right)$ to $(C, \sigma)$ where the cyclic ordering $\sigma$ is given by $\sigma\left(x_{n}\right)=x_{1}$ and $\sigma\left(x_{i}\right)=x_{i+1}$ for $i<n$. Or, in the other notation:

$$
J\left(x_{1}, \cdots, x_{n}\right)=\left[x_{1}, \cdots, x_{n}\right] .
$$

Definition 1.1 Let $\mathcal{Z}_{+}$denote the category with both linearly and cyclically ordered sets (ie, $O b\left(\mathcal{Z}_{+}\right)=O b(\mathcal{L}) \coprod O b(\mathcal{Z})$ and three kinds of morphisms:

(1) the usual morphisms (degree 1 monomorphisms) between objects of $\mathcal{Z}$,

(2) the usual morphisms (order preserving monomorphisms) between objects of $\mathcal{L}$ and

(3) a morphism $f: B \rightarrow C$ from a linearly ordered set $B$ to a cyclically ordered set $C$ is defined to be a morphism $f: J(B) \rightarrow C$ in $\mathcal{Z}$ (and composition is given by $f \circ g=f J(g): J(A) \rightarrow C$ if $g: A \rightarrow B$ is a morphism in $\mathcal{L}$ ).

There are no morphisms from $\mathcal{Z}$ to $\mathcal{L}$.

Note that $\mathcal{L}, \mathcal{Z}$ are full subcategories of $\mathcal{Z}_{+}$. A retraction $\bar{J}: \mathcal{Z}_{+} \rightarrow \mathcal{Z}$ is given by $J$ on $\mathcal{L}$ and the identity on $\mathcal{Z}$. The identity map on sets $C \rightarrow \bar{J}(C)$ is a natural transformation from the identity functor on $\mathcal{Z}_{+}$to the functor $\bar{J}$. This proves the following.

Proposition $1.2 \mathcal{Z}$ is a deformation retract of $\mathcal{Z}_{+}$.

The difference between $\mathcal{Z}$ and $\mathcal{Z}_{+}$is that $\mathcal{Z}_{+}$has a base point up to homotopy. The full subcategory of $\mathcal{L}$ in $\mathcal{Z}_{+}$is contractible and therefore serves as a homotopy base point for the category $\mathcal{Z}_{+}$. (A contraction is given by adding one point on the left then delete all the other points.)

\subsection{Homotopy type of $|\mathcal{Z}| \simeq\left|\mathcal{Z}_{+}\right|$}

Although $\mathcal{Z}_{+}$may be unfamiliar, the homotopy type of $\mathcal{Z}$ is well-known. See, eg, 9] or [6].

Theorem $1.3|\mathcal{Z}| \simeq\left|\mathcal{Z}_{+}\right| \simeq \mathbb{C} P^{\infty}$.

The universal circle bundle over $\mathbb{C} P^{\infty}$ pulls back to a circle bundle over the geometric realization $\left|\mathcal{Z}_{+}\right|$of $\mathcal{Z}_{+}$given by $S^{1}(C, \sigma)$ over $(C, \sigma)$ with or without base point. A precise construction will be given later. 


\subsection{Powers of the Euler class}

The Euler class of the universal circle bundle over $\mathcal{Z}$ is a 2 dimensional integral cohomology class $e_{\mathcal{Z}} \in H^{2}(\mathcal{Z}, \mathbb{Z})$. It is represented by a 2-cocycle which assigns an integer to every 2-simplex $A \rightarrow B \rightarrow C$ in $\mathcal{Z}$. However, the classical method is to choose a connection and integrate its curvature. This procedure, carried out in the next section, produces a rational 2 -cocycle on $\mathcal{Z}$ which represents this integral class. Since $\mathbb{C} P^{\infty}$ has no torsion in its homology, the integral class is uniquely determined by this rational cocycle.

The cocycle representing the Euler class can be described as follows. Given a 2 -simplex $A \rightarrow B \rightarrow C$ in $\mathcal{Z}$, we choose elements $a, b, c$ in $A, B, C$. The sign of $(a, b, c)$ is + if the images of $a, b, c$ in $C$ are distinct and in cyclic order. The sign is -1 if they are distinct and in reverse cyclic order. If they are not distinct then the sign is 0 . The cocycle $c_{\mathcal{Z}}(A, B, C)$ is defined to be $-\frac{1}{2}$ times the expected value of this sign.

For example, if $A=[a], B=[a, b], C=[a, c, b]$ with morphisms being inclusion maps then the probability is $\frac{1}{6}$ that distinct elements of $A, B, C$ will be chosen. The sign of $(a, b, c)$ is negative since it is an odd permutation of the given cyclic ordering of $C$ so

$$
c_{\mathcal{Z}}(A, B, C)=-\left(-\frac{1}{2}\right)\left(\frac{1}{6}\right)=+\frac{1}{12} .
$$

More generally, suppose that

$$
C_{*}=\left(C_{0} \rightarrow C_{1} \rightarrow \cdots \rightarrow C_{2 k}\right)
$$

is a $2 k$ simplex in $\mathcal{Z}$. Then the cyclic set cocycle $c_{\mathcal{Z}}^{k}$ is defined on $C_{*}$ by

$$
c_{\mathcal{Z}}^{k}\left(C_{*}\right)=\frac{(-1)^{k} k ! \sum \operatorname{sgn}\left(a_{0}, a_{1}, \cdots, a_{2 k}\right)}{(2 k) !\left|C_{0}\right| \cdots\left|C_{2 k}\right|}
$$

where the sum is taken over all $a_{i}$ in the image of $C_{i}$ in $C_{2 k}$ for $i=0, \cdots, 2 k$ and the sign of $\left(a_{0}, a_{1}, \cdots, a_{2 k}\right)$ is given by comparing this ordering with the ordering induced by the cyclic ordering of $C_{2 k}$. (The sign is zero if these elements are not distinct.)

Note that in (11) the sum is the same if we take only those $2 k+1$ tuples $\left(a_{0}, a_{1}, \cdots, a_{2 k}\right)$ where $a_{i}$ is in the image of $C_{i}$ but not in the image of $C_{i-1}$. (Otherwise, take $i$ minimal so that $a_{i} \in C_{j}$ for some $j<i$ and switch $a_{i}$ and $a_{j}$, where $j$ is minimal. This gives another summand with the opposite sign and the described operation is an involution on the set of summands that we are deleting.) 
Proposition 1.4 The cyclic set cocycle $c_{\mathcal{Z}}^{k}$ is a rational cocycle on $\mathcal{Z}$.

Proof Suppose that $C_{0} \rightarrow \cdots \rightarrow C_{2 k+1}$ is a $2 k+1$ simplex in $\mathcal{Z}$. Choose one element from each $C_{i}$ at random with equal probability. Let $a_{i}$ be the image of this element in $C_{2 k+1}$. Then the following alternating sum of the signs vanishes.

$$
\sum_{i=0}^{2 k+1}(-1)^{i} \operatorname{sgn}\left(a_{0}, \cdots, \widehat{a_{i}}, \cdots, a_{2 k+1}\right)=0
$$

This is obvious if these elements are in cyclic order in $C_{2 k+1}$. It is also clear that if two consecutive elements are reversed in the cyclic ordering then every summand in the above expression changes sign. Finally, if the elements $a_{i}$ are not distinct then all summands are zero except for two terms of opposite sign.

Since expected value is a linear function, the sum of the expected values of the summands is zero, ie, the expected value of the sign is a cocycle.

\subsection{Extension of $c_{\mathcal{Z}}^{k}$ to $\mathcal{Z}_{+}$}

For the purpose of constructing the combinatorial Miller-Morita-Mumford classes $\left[c_{\mathcal{F} \text { at }}^{k}\right]$ we only need $c_{\mathcal{Z}}^{k}$ on cyclically ordered sets. However, when we go to framed graphs we will need to extend this to $\mathcal{Z}_{+}$in such a way that it is identically zero on $\mathcal{L}$.

First we note that the pull-back along the functor $J: \mathcal{L} \rightarrow \mathcal{Z}$ of the cocycle $c_{\mathcal{Z}}^{k}$ must be a coboundary since $\mathcal{L}$ is contractible. It is in fact the coboundary of the $2 k-1$ cochain $s_{k}$ given on $C_{*}=\left(C_{0} \rightarrow \cdots \rightarrow C_{2 k-1}\right)$ by

$$
s_{k}\left(C_{*}\right)=\frac{(-1)^{k} k ! \sum \operatorname{sgn}\left(a_{0}, a_{1}, \cdots, a_{2 k-1}\right)}{(2 k) !\left|C_{0}\right| \cdots\left|C_{2 k-1}\right|} .
$$

As before, we note that this sum is unchanged if we delete terms where $a_{i} \in C_{i-1}$ for some $i$.

Definition 1.5 Given a $2 k$-simplex $C_{*}=\left(C_{0} \rightarrow \cdots \rightarrow C_{2 k}\right)$ in $\mathcal{Z}_{+}$we define $c_{\mathcal{Z}^{+}}^{k}\left(C_{*}\right)$ as follows.

(a) If the last two objects lie in $\mathcal{Z}$ we let $c_{\mathcal{Z}^{+}}^{k}\left(C_{*}\right)=c_{\mathcal{Z}}^{k} \bar{J}\left(C_{*}\right)$ where $\bar{J}: \mathcal{Z}_{+} \rightarrow$ $\mathcal{Z}$ is the retraction which is equal to $J$ on $\mathcal{L}$.

(b) If all the objects lie in $\mathcal{L}$ then we let $c_{\mathcal{Z}^{+}}^{k}\left(C_{*}\right)=0$.

(c) If $C_{2 k-1} \in \mathcal{L}$ and $C_{2 k} \in \mathcal{Z}$ then let

$$
c_{\mathcal{Z}^{+}}^{k}\left(C_{*}\right)=c_{\mathcal{Z}}^{k} \bar{J}\left(C_{*}\right)-s_{k}\left(C_{0} \rightarrow \cdots \rightarrow C_{2 k-1}\right)
$$

where $s_{k}$ is given in (2) above. 
Note that in all three cases $c_{\mathcal{Z}^{+}}^{k}\left(C_{*}\right)=0$ if the sets $C_{0}, \cdots, C_{2 k-1}$ do not have distinct cardinalities.

Since $\delta s_{k}=c_{\mathcal{Z}} \mid \mathcal{L}$ it follows that:

Proposition 1.6 The extended cyclic set cocycle $c_{\mathcal{Z}^{+}}^{k}$ is a rational $2 k$-cocycle on $\mathcal{Z}_{+}$.

In the next section we prove the following.

Theorem 1.7 The rational $2 k$-cocycle $c_{\mathcal{Z}}^{k}$ represents the $k^{\text {th }}$ power $e_{\mathcal{Z}}^{k}$ of the Euler class $e_{\mathcal{Z}} \in H^{2}(\mathcal{Z}, \mathbb{Z})$.

Since $|\mathcal{Z}| \simeq\left|\mathcal{Z}^{+}\right|$and $c_{\mathcal{Z}}^{k}=c_{\mathcal{Z}^{+}}^{k} \mid \mathcal{Z}$ we get:

Corollary 1.8 The extended cyclic set cocycle $c_{\mathcal{Z}^{+}}^{k}$ represents the $k^{\text {th }}$ power $e_{\mathcal{Z}^{+}}^{k}$ of the Euler class $e_{\mathcal{Z}^{+}}$of $\mathcal{Z}^{+}$.

\section{The curvature form on $|\mathcal{Z}|$}

In this section we obtain the cyclic $2 k$-cocycle $c_{\mathcal{Z}}^{k}$ as an elementary exercise in differential geometry. Briefly the idea is that we want to find a natural connection $A$ on the geometric realization $|\mathcal{Z}|$ of the category $\mathcal{Z}$, take the powers of the associated curvature form

$$
\Omega=d A
$$

and integrate over the even dimensional simplices of $|\mathcal{Z}|$. The same curvature form appears in [10] giving the Euler class for a space $B U(1)^{\text {comb }}$ which is closely related to $|\mathcal{Z}|$.

\subsection{Smooth families of cyclically ordered sets}

The first step is to construct a (piecewise) smooth space of cyclically ordered sets. The idea is simple. We view a cyclically ordered set with $n$ elements as being $n$ points evenly spaced on a unit circle. A smooth version of this is to divide the circle into $n$ arcs of varying length (but with constant total length $2 \pi)$. By letting the lengths of some arcs go to zero we can change the number of elements in the cyclically ordered set in a continuous way. 
We define the weight of an arc to be its length divided by $2 \pi$. Then, up to rotation, an element in this space is represented by a cyclically ordered sequence of $n$ nonnegative real numbers $\left[w_{1}, \cdots, w_{n}\right]$ whose sum is 1 . We make this precise:

Definition 2.1 A cyclic weight system is a triple $(C, \sigma, w)$ where $(C, \sigma)$ is a cyclically ordered set and $w: C \rightarrow I$ is a nonnegative real valued function on $C$ so that

$$
\sum_{x \in C} w(x)=1
$$

Given a cyclic weight system $(C, \sigma, w)$, the canonical circle over $(C, \sigma, w)$ is given by

$$
S^{1}(C, \sigma, w)=\coprod_{x \in C} x \times[0, w(x)] / \sim
$$

where the identifications are given by $(x, w(x)) \sim(\sigma(x), 0)$, ie, the line segments $x \times[0, w(x)]$ are connected end to end in a circle. If $w_{t}, t \in P$, is a smooth family of weights on a fixed cyclically ordered set $(C, \sigma)$ then we can form a smooth principal $S^{1}$-bundle over $P$ by:

$$
S^{1}\left(C, \sigma, w_{P}\right)=\coprod_{x \in C}\left\{(s, t) \in I \times P \mid s \leq w_{t}(x)\right\} / \sim
$$

with the fiberwise identification $\left(x, w_{t}(x), t\right) \sim(\sigma(x), 0, t)$.

If we choose a starting point, the elements of $C$ can be written $\left(x_{1}, \cdots, x_{n}\right)$ (with cyclic order $\left[x_{1}, \cdots, x_{n}\right]$ ) and we can write $w_{j}=w\left(x_{j}\right)$. If we place line segments of length $w_{j}$ next to each other in sequence on the real line starting at the origin then the center of mass of the $j^{\text {th }}$ segment will be located at a point

$$
s_{j}=w_{1}+\cdots+\omega_{j-1}+\frac{1}{2} w_{j}
$$

units from the starting point. On the circle this will be the point $\exp \left(2 \pi i s_{j}\right)$.

The angular momentum of the circle is then given by:

$$
\sum w_{j} \exp \left(-2 \pi i s_{j}\right) d \exp \left(2 \pi i s_{j}\right)=2 \pi i \sum w_{j} d s_{j} .
$$

This means that, with respect to the inertial frame, our coordinate system is rotating in the other direction at this rate. Consequently the inertial connection (in this coordinate frame) is given by

$$
A=-2 \pi i \sum w_{j} d s_{j}=-2 \pi i \sum_{1 \leq i<j \leq n} w_{j} d w_{i}-\pi i \sum w_{j} d w_{j} .
$$


The curvature of this form is

$$
\Omega=d A=d A=2 \pi i \sum_{1 \leq i<j \leq n} d w_{i} \wedge d w_{j} .
$$

Therefore, the Euler class of the canonical circle bundle over the space of cyclic weight systems is given by the differential form

$$
\frac{i}{2 \pi} \Omega=-\sum_{1 \leq i<j \leq n} d w_{i} \wedge d w_{j}
$$

This is invariant under cyclic permutation of the $w_{j}$ since the summands with $i=1$ add up to $d w_{1} \wedge\left(d w_{2}+\cdots+d w_{n}\right)=d w_{1} \wedge\left(-d w_{1}\right)=0$. Similarly, the terms with $j=n$ add up to zero.

We interpret the "space of cyclic weight systems" to be the geometric realization $|\mathcal{Z}|$ of $\mathcal{Z}$.

In [10] Konsevich obtained (4) (with all terms having $j=n$ deleted) as the Euler class on a space $B U(1)^{\text {comb }}$ which he defined to be the space of isomorphism classes of cyclic weight systems. If $\mathcal{Z}_{0}$ is the full subcategory of $\mathcal{Z}$ given by choosing one object from each isomorphism class then we get a quotient map $\left|\mathcal{Z}_{0}\right| \rightarrow B U(1)^{c o m b}$ which is a rational homotopy equivalence. Kontsevich shows that the Euler class of $B U(1)^{c o m b}$ is given by a 2 -form $\omega$. The differential form (4I) is the pull-back of this 2 -form.

\subsection{Simplices in $|\mathcal{Z}|$}

Strictly speaking $|\mathcal{Z}|$ is the geometric realization of the simplicial nerve $\mathcal{N}_{\bullet}(\mathcal{Z})$ of $\mathcal{Z}$. A $p$-simplex

$$
C_{*}=\left(C_{0} \rightarrow C_{1} \rightarrow \cdots \rightarrow C_{p}\right)
$$

in $\mathcal{Z}$ is one element of $\mathcal{N}_{p}(\mathcal{Z})$ but it represents a geometric $p$-simplex

$$
\Delta^{p} \times\left\{C_{*}\right\} \subseteq|\mathcal{Z}|
$$

By definition, $|\mathcal{Z}|$ is the union of these geometric simplices:

$$
|\mathcal{Z}|=\coprod_{x \in \mathcal{N}_{p}(\mathcal{Z}), p \geq 0} \Delta^{p} \times x / \sim
$$

The vertices $v_{0}, \cdots, v_{p}$ of $\Delta^{p}$ correspond to the cyclic sets $C_{0}, \cdots, C_{p}$. The other points $t \in \Delta^{p}$ correspond to cyclic weight systems given by mass functions on $C_{p}$. The set $C_{j}$ is identified with the mass function on $C_{p}$ which is 1 on the image of $C_{j}$ and 0 in the complement. For simplicity of notation we will identify $C_{j}$ with its image in $C_{p}$. 
We parametrize the $p$-simplex $\Delta^{p}$ by

$$
\Delta^{p}=\left\{t \in \mathbb{R}^{p} \mid 1 \geq t_{1} \geq t_{2} \geq \cdots \geq t_{p} \geq 0\right\}
$$

and we take $t_{0}=1, t_{p+1}=0$ to be fixed. Then the cyclic weight system $C(t)$ for $t \in \Delta^{p}$ will be given by the mass function

$$
\mu_{t}: C_{p} \rightarrow I
$$

given by $\mu_{t}(a)=t_{j}$ if $a \in C_{j}-C_{j-1}$. The weights are the normalized masses

$$
w_{t}(a)=\frac{\mu_{t}(a)}{M_{t}}=\frac{\mu_{t}(a)}{\sum \mu_{t}(a)}
$$

where $M_{t}=\sum \mu_{t}(a)$ is the total mass. The weights are ordered according to the cyclic ordering of the elements of $C_{p}$.

The canonical circle bundle $E_{\mathcal{Z}}$ over $|\mathcal{Z}|$ is given by

$$
E_{\mathcal{Z}}=\coprod_{x \in \mathcal{N}_{p}(\mathcal{Z}), p \geq 0} S^{1}\left(C_{p}, w_{\Delta^{p}}\right) \times x / \sim
$$

Note that each piece $S^{1}\left(C_{p}, w_{\Delta^{p}}\right) \times x$ is a smooth principal $S^{1}$ bundle over $\Delta^{p} \times x$. Consequently, $E_{\mathcal{Z}}$ is a piecewise smooth principal $S^{1}$-bundle over $|\mathcal{Z}|$.

The $j^{\text {th }}$ vertex $v_{j}$ of $\Delta^{p}$ is given by

$$
t_{0}=t_{1}=\cdots=t_{j}=1, \quad t_{j+1}=\cdots=t_{p}=t_{p+1}=0 .
$$

This agrees with the discussion above since it assigns a mass of 1 to the elements of $C_{j}$. The barycentric coordinates on $\Delta^{p}$ are given by $t_{j}^{\prime}=t_{j}-t_{j+1}$ so that the $j^{\text {th }}$ face is given by $t_{j}=t_{j+1}$.

\subsection{The Euler class on 2 -simplices}

Now take $p=2$. Take a fixed 2-simplex $C_{0} \rightarrow C_{1} \rightarrow C_{2}$. Let $a=\left|C_{0}\right|, b=$ $\left|C_{1}\right|-a$ and $c=\left|C_{2}\right|-a-b$. Denote the elements of $C_{2}$ in cyclic order by $\left(x_{1}, x_{2}, \cdots, x_{n}\right)$ where $n=a+b+c$.

If $t=\left(t_{1}, t_{2}\right) \in \Delta^{2}$ then the mass function $\mu_{t}$ is given by

$$
\mu_{t}\left(x_{i}\right)= \begin{cases}t_{0}=1 & \text { if } x_{i} \in C_{0}, \\ t_{1} & \text { if } x_{i} \in C_{1}-C_{0}, \\ t_{2} & \text { if } x_{i} \in C_{2}-C_{1} .\end{cases}
$$

We note that $t_{0}$ occurs $a$ times, $t_{1}$ occurs $b$ times and $t_{2}$ occurs $c$ times. Thus the total mass is

$$
M_{t}=\sum \mu_{t}\left(x_{i}\right)=a+b t_{1}+c t_{2}
$$


The weight (relative mass) of $x_{i}$ is

$$
w_{t}\left(x_{i}\right)=\frac{\mu_{t}\left(x_{i}\right)}{M_{t}}=\frac{t_{j}}{a+b t_{1}+c t_{2}}
$$

where $j=0,1$ or 2 .

Theorem 2.2 The 2-cocycle on $\mathcal{Z}$ whose value on the 2 -simplex $\left(C_{0} \rightarrow\right.$ $C_{1} \rightarrow C_{2}$ ) is given by

$$
\int_{\Delta^{2}} \frac{i}{2 \pi} \Omega=-\int_{1 \geq t_{1} \geq t_{2} \geq 0} \sum_{1 \leq i<j \leq n} d w_{t}\left(x_{i}\right) \wedge d w_{t}\left(x_{j}\right)
$$

is equal to the $2-$ cocyle $c_{\mathcal{Z}}$ of section 1.4

Proof Up to sign there are only three possibilities for the 2 -form $d w_{t}\left(x_{i}\right) \wedge$ $d w_{t}\left(x_{j}\right)$ :

$$
\begin{aligned}
& d\left(\frac{1}{M_{t}}\right) \wedge d\left(\frac{t_{1}}{M_{t}}\right)=-\frac{d M_{t}}{M_{t}^{2}} \wedge \frac{d t_{1}}{M_{t}}=\frac{-c d t_{2} \wedge d t_{1}}{M_{t}^{3}}=c \frac{d t_{1} \wedge d t_{2}}{M_{t}^{3}} \\
& d\left(\frac{1}{M_{t}}\right) \wedge d\left(\frac{t_{2}}{M_{t}}\right)=-\frac{d M_{t}}{M_{t}^{2}} \wedge \frac{d t_{2}}{M_{t}}=-b \frac{d t_{1} \wedge d t_{2}}{M_{t}^{3}} \\
& d\left(\frac{t_{1}}{M_{t}}\right) \wedge d\left(\frac{t_{2}}{M_{t}}\right)=\left[1-\frac{b t_{1}}{M_{t}}-\frac{c t_{2}}{M_{t}}\right] \frac{d t_{1} \wedge d t_{2}}{M_{t}^{2}}=a \frac{d t_{1} \wedge d t_{2}}{M_{t}^{3}}
\end{aligned}
$$

We interpret (5) as a sum of $c$ terms (one for each $x_{k} \in C_{2}-C_{1}$ ) and similarly for (6) and (7). Then for every triple of indices $(i, j, k)$ so that $x_{i} \in C_{0}$, $x_{j} \in C_{1}-C_{0}$ and $x_{k} \in C_{2}-C_{1}$ we get three terms, one of each kind, adding up to:

$$
(\operatorname{sgn}(j-i)-\operatorname{sgn}(k-i)+\operatorname{sgn}(k-j)) \frac{d t_{1} \wedge d t_{2}}{M_{t}^{3}}
$$

A little thought will show that the sum of signs is 1 if $i, j, k$ are in cyclic order and -1 if not. Ie,

$$
\operatorname{sgn}(j-i)-\operatorname{sgn}(k-i)+\operatorname{sgn}(k-j)=\operatorname{sgn}(i, j, k) .
$$

Furthermore, we have the easy double integral:

$$
\int_{1 \geq t_{1} \geq t_{2} \geq 0} \frac{d t_{1} d t_{2}}{\left(a+b t_{1}+c t_{2}\right)^{3}}=\frac{1}{2 a(a+b)(a+b+c)} .
$$

Putting these together we get:

$$
\int_{\Delta^{2}} \frac{i}{2 \pi} \Omega=-\sum_{i, j, k \in C_{2}} \frac{\operatorname{sgn}(i, j, k)}{2 a(a+b)(a+b+c)}=c_{\mathcal{Z}}\left(C_{0} \rightarrow C_{1} \rightarrow C_{2}\right)
$$




\section{$2.4 e_{\mathcal{Z}}^{k}$ on $2 k-$ simplices}

Now take $p=2 k$. Let $C_{0} \rightarrow \cdots \rightarrow C_{2 k}$ be a $2 k$-simplex in $\mathcal{Z}$. Let $a_{0}=\left|C_{0}\right|$ and $a_{j}=\left|C_{j}\right|-\left|C_{j-1}\right|$ for $j \geq 1$. Let $\left(x_{1}, \cdots, x_{n}\right)$ denote the elements of $C_{2 k}$ in cyclic order.

The mass function $\mu_{t}$ for $t \in \Delta^{2 k}$ is given by $\mu_{t}\left(x_{i}\right)=t_{j}$ if $x_{i} \in C_{j}-C_{j-1}$. Then the total mass is

$$
M_{t}=\sum_{i=1}^{n} \mu_{t}\left(x_{i}\right)=\sum_{j=0}^{2 k} a_{j} t_{j}
$$

The weight of $x_{i}$ is

$$
w_{t}\left(x_{i}\right)=\frac{\mu_{t}\left(x_{i}\right)}{M_{t}}=\frac{t_{j}}{a_{0} t_{0}+\cdots+a_{2 k} t_{2 k}}
$$

for some $j$.

A cocycle for $e_{\mathcal{Z}}^{k}$ is given on $2 k$-simplices by integrating the $2 k$-form $\left(\frac{i}{2 \pi} \Omega\right)^{k}$ over $\Delta^{2 k}$. This form can be expanded:

$$
\begin{aligned}
\left(\frac{i}{2 \pi} \Omega\right)^{k} & =(-1)^{k} \sum_{i_{1}<j_{1}} \cdots \sum_{i_{k}<j_{k}} d w_{t}\left(x_{i_{1}}\right) \wedge d w_{t}\left(x_{j_{1}}\right) \wedge \cdots \wedge d w_{t}\left(x_{i_{k}}\right) \wedge d w_{t}\left(x_{j_{k}}\right) \\
& =(a)(-1)^{k} k ! \sum_{i_{1}<i_{2}<\cdots<i_{k}} \sum_{i_{1}<j_{1}} \cdots \sum_{i_{k}<j_{k}} d w_{t}\left(x_{i_{1}}\right) \wedge \cdots \wedge d w_{t}\left(x_{j_{k}}\right) \\
& =(b)(-1)^{k} k ! \sum_{i_{1}<j_{1}<i_{2}<\cdots<i_{k}<j_{k}} d w_{t}\left(x_{i_{1}}\right) \wedge \cdots \wedge d w_{t}\left(x_{j_{k}}\right) \\
& =(-1)^{k} k ! \sum_{i_{1}<i_{2}<\cdots<i_{2 k}} d w_{t}\left(x_{i_{1}}\right) \wedge \cdots \wedge d w_{t}\left(x_{i_{2 k}}\right)
\end{aligned}
$$

where (a) is by symmetry and (b) follows from the fact that the summands in the second line not in the third come in cancelling pairs: Take the first $j_{p}>i_{p+1}$ and switch it with $j_{p+1}$.

Suppose that $w_{t}\left(x_{i_{p}}\right)=t_{j_{p}} / M_{t}$. Then there are basically only two possibilities for $d w_{t}\left(x_{i_{1}}\right) \wedge \cdots \wedge d w_{t}\left(x_{i_{2 k}}\right)$ :

(a) If $j_{p} \neq 0$ for all $p$ then

$$
\begin{aligned}
& d w_{t}\left(x_{i_{1}}\right) \wedge \cdots \wedge d w_{t}\left(x_{i_{2 k}}\right)=\operatorname{sgn}\left(j_{1}, \cdots, j_{2 k}\right) d\left(\frac{t_{1}}{M_{t}}\right) \wedge \cdots \wedge d\left(\frac{t_{2 k}}{M_{t}}\right) \\
& =\operatorname{sgn}\left(j_{1}, \cdots, j_{2 k}\right)\left(1-\frac{a_{1} t_{1}}{M_{t}}-\cdots-\frac{a_{2 k} t_{2 k}}{M_{t}}\right) \frac{d t_{1} \wedge d t_{2} \wedge \cdots \wedge d t_{2 k}}{M_{t}^{2 k}}
\end{aligned}
$$




$$
=a_{0} \operatorname{sgn}\left(0, j_{1}, \cdots, j_{2 k}\right) \frac{d t_{1} \wedge d t_{2} \wedge \cdots \wedge d t_{2 k}}{M_{t}^{2 k+1}}
$$

(b) If $j_{p}=0$ for some $p$ then the corresponding weight is $w_{t}\left(x_{i_{p}}\right)=\frac{1}{M_{t}}$ and we have $d\left(\frac{1}{M_{t}}\right)$ instead of $d\left(\frac{t_{q}}{M_{t}}\right)$ for some $q$. This means we should replace the term $d\left(\frac{1}{M_{t}}\right)$ by $-\frac{a_{q} d t_{q}}{M_{t}^{2}}$ and we get:

$$
d w_{t}\left(x_{i_{1}}\right) \wedge \cdots \wedge d w_{t}\left(x_{i_{2 k}}\right)=a_{q} \operatorname{sgn}\left(q, j_{1}, \cdots, j_{2 k}\right) \frac{d t_{1} \wedge \cdots \wedge d t_{2 k}}{M_{t}^{2 k+1}}
$$

As before, we interpret (a) as a sum of $a_{0}$ terms, one for each element of $C_{0}$, and similarly for (b). Then for every choice of $x_{i_{0}} \in C_{0}, x_{i_{1}} \in C_{1}-C_{0}, \cdots, x_{i_{2 k}} \in$ $C_{2 k}-C_{2 k-1}$ we get the following.

$$
\sum_{q=0}^{2 k} \operatorname{sgn}\left(x_{i_{q}}, x_{i_{0}}, \cdots, \widehat{x_{i_{q}}}, \cdots, x_{i_{2 k}}\right) \frac{d t_{1} \wedge \cdots \wedge d t_{2 k}}{M_{t}^{2 k+1}}
$$

The permutations in (10) are the inverses of the permutations in (a),(b) above so they have the same sign.

The summands in (10) are equal with alternating signs. Since there are an odd number of terms it is equal to its first summand. The $2 k$-form $\left(1 / M_{t}^{2 k+1}\right) d t_{1} \wedge$ $\cdots \wedge d t_{2 k}$ has integral:

$$
\begin{aligned}
\int_{\Delta^{2 k}} \frac{d t_{1} \wedge \cdots \wedge d t_{2 k}}{M_{t}^{2 k+1}} & =\int_{1 \geq t_{1} \geq \cdots \geq t_{2 k} \geq 0} \frac{d t_{1} \cdots d t_{2 k}}{\left(a_{0}+a_{1} t_{1}+\cdots+a_{2 k} t_{2 k}\right)^{2 k+1}} \\
& =\frac{1}{(2 k) ! a_{0}\left(a_{0}+a_{1}\right) \cdots\left(a_{0}+\cdots+a_{2 k}\right)}
\end{aligned}
$$

which is an easy induction on $2 k$.

Combining this with the formula for $\left(\frac{i}{2 \pi} \Omega\right)^{k}$ we get:

Theorem 2.3 The integral of $\left(\frac{i}{2 \pi} \Omega\right)^{k}$ over the $2 k$-simplex $\Delta^{2 k} \times\left\{C_{*}\right\}$ is

$$
\int_{\Delta^{2 k}}\left(\frac{i}{2 \pi} \Omega\right)^{k}=(-1)^{k} k ! \frac{\sum \operatorname{sgn}\left(x_{i_{0}}, \cdots, x_{i_{2 k}}\right)}{(2 k) !\left|C_{0}\right| \cdots\left|C_{2 k}\right|}
$$

where the sum is taken over all $x_{i_{0}} \in C_{0}, x_{i_{1}} \in C_{1}-C_{0}, \cdots, x_{i_{2 k}} \in C_{2 k}-C_{2 k-1}$.

In other words, the deRham cocycles for the powers of the Euler class on $|\mathcal{Z}|$ are equal to the combinatorial cocycles $c_{\mathcal{Z}}^{k}$. 


\section{Combinatorial formula for MMM classes}

We construct cocycles $c_{\mathcal{F} \text { at }}^{k}$ on the category of fat graphs $\mathcal{F}$ at by evaluating the cyclic set cocycles $c_{\mathcal{Z}}^{k}$ on each vertex. The classifying space of this category is well-known to be homotopy equivalent to the disjoint union of classifying spaces of mapping class groups $M_{g}^{s}$ of surfaces of genus $g$ with $s \geq \max (1,3-2 g)$ punctures.

$$
|\mathcal{F} a t| \simeq \coprod_{s \geq \max (1,3-2 g)} B M_{g}^{s}
$$

(This is Theorem 3.1 below.) Thus a cohomology class for $\mathcal{F}$ at gives a cohomology class for each mapping class group $M_{g}^{s}$.

By direct computation we show that the cohomology classes $\left[c_{\mathcal{F} \text { at }}^{k}\right]$ are dual to the Witten cycles $W_{k}$. More precisely,

$$
\left[c_{\mathcal{F} a t}^{k}\right]=(-1)^{k} \frac{k !}{(2 k+1) !}\left[W_{k}\right]^{*} .
$$

In Theorem 5.1 below we will show that $\left[c_{\mathcal{F} a t}^{k}\right]=-2 \widetilde{\kappa}_{k}$. So the adjusted fat graph cocycle $-\frac{1}{2} c_{\mathcal{F} \text { at }}^{k}$ is a combinatorial formula for $\widetilde{\kappa}_{k}$.

\subsection{The category of fat graphs}

We define a fat graph to be a finite connected graph $\Gamma$ possibly with loops and multiple edges in which every vertex has valence $\geq 3$ together with a cyclic ordering of the edges incident to each vertex. To be precise and to fix our notation, the fat graph $\Gamma$ consists of

(1) $\Gamma^{0}$, the set of vertices,

(2) $\Gamma^{\frac{1}{2}}$, the set of half edges,

(3) $\partial: \Gamma^{\frac{1}{2}} \rightarrow \Gamma^{0}$, the incidence or boundary map so that $\left|\partial^{-1}(v)\right| \geq 3$ for all vertices $v$,

(4) $\sigma$, a cyclic ordering on each set $\partial^{-1}(v)$ and

(5) $a \mapsto \bar{a}$, a fixed-point free involution on $\Gamma^{\frac{1}{2}}$ whose orbits we call edges.

Note that each edge $\{a, \bar{a}\}$ has two orientations $e=(a, \bar{a})$ and $\bar{e}=(\bar{a}, a)$. Each oriented edge $e=(a, b)$ has a source $s(e)=\partial a$ and target $t(e)=\partial b$.

For several reasons we need to consider the cyclically ordered set of "angles" between incident half edges at each vertex of a fat graph. An angel (at $v$ ) is 
defined to be an ordered pair of half edges $(a, b)$ so that $\partial a=\partial b=v$ and $b=\sigma(a)$. In other words, $a, b$ are incident to the same vertex $v$ and $b$ is one step counterclockwise from $a$. Let $C(v)$ be the set of angles at $v$. Then $C(v)$ has a cyclic ordering $\sigma(a, b)=(b, \sigma(b))$.

For example, the figure " $\infty$ " has one vertex $v$ of valence 4 with $\partial^{-1}(v)=$ $[a, \bar{a}, b, \bar{b}]$ and $C(v)=[(a, \bar{a}),(\bar{a}, b),(b, \bar{b}),(\bar{b}, a)]$. As before, we denote cyclically ordered sets by square brackets.

A fat graph $\Gamma$ is evidently equal to the core of some connected oriented punctured surface $\Sigma_{\Gamma}$ which is well-defined up to homeomorphism.

A morphism $f: \Gamma_{1} \rightarrow \Gamma_{2}$ of fat graphs is a morphism of graphs where the inverse image of every open edge is an open edge and the inverse image of every vertex is a tree in $\Gamma_{1}$ with the cyclic ordering of the half edges incident to each vertex of $\Gamma_{2}$ corresponds to the cyclic ordering of the half edges incident to the corresponding tree in $\Gamma_{1}$. (In other words, the surfaces are homeomorphic.)

The codimension of a graph is defined to be the non-negative integer

$$
\operatorname{codim} \Gamma=\sum(\operatorname{val}(v)-3) .
$$

Thus, $\operatorname{codim} \Gamma=0$ if and only if $\Gamma$ is trivalent. It is important to note that, for any morphism $f: \Gamma_{1} \rightarrow \Gamma_{2}$ which is not an isomorphism, codim $\Gamma_{1}>\operatorname{codim} \Gamma_{2}$.

The category of all fat graphs will be denoted $\mathcal{F}$ at. Since the punctured surface $\Sigma_{\Gamma}$ is fixed up to homeomorphism on each component of $\mathcal{F}$ at we have:

$$
\mathcal{F} a t=\coprod \mathcal{F} a t_{g}^{s}
$$

where $\mathcal{F} a t_{g}^{s}$ is the full subcategory of fat graphs $\Gamma$ so that $\Sigma_{\Gamma}$ is a surface of genus $g$ with $s$ punctures.

There is a well-known correspondence between fat graphs, the moduli space of curves and the mapping class group. In the present context it says the following.

Theorem 3.1 (Penner [17, Strebel [20]) The geometric realization of the category $\mathcal{F}_{a t}^{s}$ is homotopy equivalent to the classifying space of the mapping class group $M_{g}^{s}$ of genus $g$ surfaces with $s$ punctures provided that $\mathcal{F} a t_{g}^{s}$ is non-empty, ie, $s \geq 1$ and $s+2 g \geq 3$.

Proof (For a more detailed proof see [7, Theorem 8.6.3.) Let $S$ be a fixed oriented surface of genus $g$ with $s$ boundary components. Then, by a theorem of Culler and Vogtmann [2], the space of all pairs $(\Gamma, f)$ where $\Gamma$ is a fat graph (an element of $|\mathcal{F} a t|$ ) and $f$ is an orientation preserving homeomorphism $f: \Sigma_{\Gamma} \rightarrow S$ is contractible and $\mathrm{Homeo}_{+}(S)$ acts freely on this space with quotient $\left|\mathcal{F} a t_{g}^{s}\right|$. Thus $\left|\mathcal{F} a t_{g}^{s}\right| \simeq B$ Homeo+ $_{+}(S) \simeq B M_{g}^{s}$. 


\subsection{The fat graph cocycle $c_{\mathcal{F} a t}^{k}$}

For each vertex $v$ of $\Gamma_{1}$, a morphism $f: \Gamma_{1} \rightarrow \Gamma_{2}$ sends the angle set $C(v)$ monomorphically into $C(f(v))$ in a cyclic order preserving way. Thus to a $2 k$-simplex

$$
\Gamma_{*}=\left(\Gamma_{0} \rightarrow \Gamma_{1} \rightarrow \cdots \rightarrow \Gamma_{2 k}\right)
$$

in the nerve of $\mathcal{F}$ at we can extract several $2 k$-simplices in the nerve of $\mathcal{Z}$, one for each vertex of $\Gamma_{0}$.

Definition 3.2 Let $c_{\mathcal{F} a t}^{k}$ be the $2 k$-cochain on $\mathcal{F}$ at given by

$$
c_{\mathcal{F} a t}^{k}\left(\Gamma_{*}\right)=\sum_{v \in \Gamma_{0}^{0}} m(v) c_{\mathcal{Z}}^{k}\left(C(v) \rightarrow C\left(f_{1}(v)\right) \rightarrow \cdots \rightarrow C\left(f_{2 k}(v)\right)\right)
$$

where $m(v)=\operatorname{val}(v)-2$ is the multiplicity of $v$ and $f_{i}=f_{i 0}: \Gamma_{0} \rightarrow \Gamma_{i}$ is the composition

$$
f_{i 0}: \Gamma_{0} \stackrel{f_{10}}{\longrightarrow} \Gamma_{1} \stackrel{f_{21}}{\longrightarrow} \cdots \stackrel{f_{i-1 i}}{\longrightarrow} \Gamma_{i}
$$

of arrows in $\Gamma_{*}$.

Every time an edge collapses, two half edges disappear. Consequently, the multiplicity of the resulting vertex is the sum of the multiplicities of the original two vertices. More generally, we have:

Lemma 3.3 Given any morphism $f: \Gamma_{1} \rightarrow \Gamma_{2}$ in $\mathcal{F}$ at and any vertex $v$ in $\Gamma_{2}$ we have:

$$
m(v)=\sum_{w \in f^{-1}(v)} m(w),
$$

ie, the multiplicity of $v$ is the sum of the multiplicities of the vertices which collapsed to $v$.

Proof This follows from the fact that $T=f^{-1}(v)$ is a tree. Thus $T$ has $n$ edges and $n+1$ vertices $w_{0}, \cdots, w_{n}$. So,

$$
m(v)=\operatorname{val}(v)-2=\sum \operatorname{val}\left(w_{i}\right)-2 n-2=\sum m\left(w_{i}\right) .
$$

Theorem $3.4 c_{\mathcal{F} a t}^{k}$ is a rational $2 k$-cocycle on $\mathcal{F}$ at which determines a welldefined integral cohomology class

$$
\left[c_{\mathcal{F} \text { at }}^{k}\right] \in H^{2 k}(\mathcal{F} a t, \mathbb{Z}) .
$$


Proof Given any $2 k+1$-simplex $\Gamma_{*}=\left(\Gamma_{0}, \cdots, \Gamma_{2 k+1}\right)$ we have:

$$
\begin{aligned}
\delta c_{\mathcal{F} a t}^{k}\left(\Gamma_{*}\right) & =\sum_{i=0}^{2 k+1}(-1)^{i} c_{\mathcal{F} a t}^{k}\left(\Gamma_{0}, \cdots, \widehat{\Gamma}_{i}, \cdots, \Gamma_{2 k+1}\right) \\
& =\sum_{v_{1} \in \Gamma_{1}^{0}} m\left(v_{1}\right) c_{\mathcal{Z}}^{k}\left(C\left(v_{1}\right), \cdots, C\left(v_{2 k+1}\right)\right) \\
& +\sum_{i=1}^{2 k+1}(-1)^{i} \sum_{v_{0} \in \Gamma_{0}^{0}} m\left(v_{0}\right) c_{\mathcal{Z}}^{k}\left(C\left(v_{0}\right), \cdots, \widehat{C\left(v_{i}\right)}, \cdots, C\left(v_{2 k+1}\right)\right)
\end{aligned}
$$

where the $v_{i}$ are related by $v_{i}=f_{i j}\left(v_{j}\right)$ for all $j<i$. Since $c_{\mathcal{Z}}^{k}$ is a cocycle, the last sum is equal to

$$
-\sum_{v_{0} \in \Gamma_{0}^{0}} m\left(v_{0}\right) c_{\mathcal{Z}}^{k}\left(C\left(v_{1}\right), \cdots, C\left(v_{2 k+1}\right)\right)
$$

which exactly cancels the second sum by Lemma 3.3. Thus $c_{\mathcal{F} a t}^{k}$ is a (rational) cocycle. But the above argument uses only the fact that $c_{\mathcal{Z}}^{k}$ is a $2 k$-cycle on the category $\mathcal{Z}$. Therefore we may replace $c_{\mathcal{Z}}^{k}$ with an integral cocycle. Since $|\mathcal{Z}| \simeq \mathbb{C} P^{\infty}$ has no torsion in its homology, this integral class is well defined up to an integral coboundary so the same holds for $c_{\mathcal{F} a t}^{k}$.

The simplest example is $k=0$. Then

$$
c_{\mathcal{F} a t}^{0}(\Gamma)=\sum_{v \in \Gamma^{0}} m(v)=-2 \chi(\Gamma)=-2 \chi\left(\Sigma_{\Gamma}\right),
$$

ie, negative 2 times the Euler characteristic of $\Gamma \simeq \Sigma_{\Gamma}$.

\subsection{Smooth families of fat graphs}

Suppose we have a smooth family of punctured surfaces, ie, a smooth bundle $\Sigma \rightarrow E \stackrel{p}{\rightarrow} M$ where $M$ is a compact smooth $n-$ manifold with a fixed trivialization $E \mid \partial M=\Sigma \times \partial M$ over the boundary $\partial M$ of $M$. If $\Sigma$ is an oriented surface of genus $g$ with $s \geq 1$ boundary components then $p: E \rightarrow M$ is classified by a continuous mapping

$$
f:(M, \partial M) \rightarrow\left(B M_{g}^{s}, *\right) .
$$

By the simplicial approximation theorem we can choose any small triangulation of $(M, \partial M)$ and approximate $f$ by a simplicial map

$$
F:(T(M), T(\partial M)) \rightarrow \mathcal{N}_{\bullet} \mathcal{F} a t_{g}^{s}
$$


where $\mathcal{N}_{\bullet} \mathcal{F} a t_{g}^{s}$ is the simplicial nerve of the category $\mathcal{F} a t_{g}^{s}$ (fat graphs $\Gamma$ whose surfaces $\Sigma_{\Gamma}$ have genus $g$ wand $s$ boundary components). The following lemma implies that $F$ can be chosen so that its image contains no fat graphs of codimension $>n$.

Lemma 3.5 Let $\Gamma$ be any fat graph of codimension $c$. Then the full subcategory of $\mathcal{F}$ at $/ \Gamma$ consisting of graphs of codimension $<c$ is homotopy equivalent to a $(c-1)$-sphere.

Remark 3.6 In any $k$ simplex $\left(\Gamma_{0} \rightarrow \cdots \rightarrow \Gamma_{k}\right) \in \mathcal{N}_{k} \mathcal{F}$ at, the last object $\Gamma_{k}$ has the largest codimension. Therefore the subcategory of $\mathcal{F} a t / \Gamma$ described in the lemma is the link at $\Gamma$ of the space of fat graphs of codimension $\geq c$.

Proof Suppose $v_{1}, \cdots, v_{r}$ are the vertices of $\Gamma$ of codim $>0$. Let $c_{i}=$ $\operatorname{codim}\left(v_{i}\right)$. Then $c=\sum c_{i}$. If $\Gamma^{\prime}$ is a fat graph of codimension $c^{\prime}<c$ which maps to $\Gamma$ then in $\Gamma^{\prime}$ the vertices $v_{1}, \cdots, v_{r}$ must resolve into planar trees of codimension $c_{i}^{\prime}$ where $c_{i}^{\prime} \leq c_{i}$ and $\sum c_{i}^{\prime}=c^{\prime}<c$. In other words, $\Gamma^{\prime}$ lies on the boundary of the product

$$
A_{c_{1}+3}\left(v_{1}\right) \times \cdots \times A_{c_{r}+3}\left(v_{r}\right)
$$

of the Stasheff polyhedra $A_{c_{i}+3}\left(v_{i}\right)$ associated with the vertices $v_{i}$. But each Stasheff polyhedron is a disk so the product (14) is a disk of dimension $\sum c_{i}=c$ and $\Gamma^{\prime}$ lies on the boundary of this disk, ie, it lies on a sphere of dimension $c-1$. (Actually, $\mathcal{F} a t / \Gamma$ is much larger since it contains infinitely many isomorphic copies of each object so we get only a homotopy equivalence with $S^{c-1}$.)

Proposition 3.7 We can choose the triangulation $(T(M), T(\partial M))$ and the simplicial map $F$ so that:

(1) The image of $F$ contains no fat graphs of codimension $>n$.

(2) If a vertex $v$ of $T(M)$ maps to a fat graph of codimension $n$ then the star of $v$ maps isomorphically to the product of Stasheff polyhedra (14).

Proof By the lemma we may assume (1) and the condition that only isolated vertices $v$ of $T(M)$ map to fat graphs of codimension $n$. Then the link of such a vertex $v$ maps to the geometric realization of the subcategory $\mathcal{C}$ of $\mathcal{F} a t / F(v)$ of graphs of codimension $<n$ which is equivalent to an $n-1$ sphere by the lemma. Consequently we have a well defined degree, say $d$. Now modify the triangulation in the star of $v$ to include $d$ copies of the $n$-disk (14). The complement maps to $|\mathcal{C}|$. 


\subsection{The Witten cycle $W_{k}$}

Let $\mathcal{W}_{k}$ denote the full subcategory of $\mathcal{F}$ at consisting of fat graphs having a vertex of valence $\geq 2 k+3$. Then it is well-known that the realization

$$
W_{k}=\left|\mathcal{W}_{k}\right|
$$

is a codimension $2 k$ subset of $|\mathcal{F} a t|$ whose dual $\left[W_{k}\right]^{*}$ is a well-defined cohomology class for the mapping class group. In the original finite dimensional model of $\left[18\right.$ and $\left[10,\left[W_{k}\right]^{*}\right.$ is the Poincaré dual of the properly embedded suborbifold $W_{k}$. For the category $\mathcal{F} a t$, we need to rely on Proposition 3.7 Given any smooth family of punctured surfaces $\Sigma \rightarrow E \rightarrow M$ as above where $n=\operatorname{dim} M=2 k$, we use Proposition 3.7 to make $M$ "transverse" to $W_{k}$. A finite number of vertices in the triangulation $T(M)$ will map to the Witten cycle $W_{k}$. We count these with the orientation convention of Definition 3.11] below. To see directly that this gives a well-defined integer we need to show that $\left[W_{k}\right]^{*}$ is zero on the link of a codimension $2 k+1$ graph. Penner shows this geometrically in 18. In this paper this will be a consequence of our calculations since we prove that $\left[c_{\mathcal{F} a t}^{k}\right]$ is dual to the cycle $\left[W_{k}\right]$. The following theorem implies that the signed intersection number of a $2 k$ parameter family of fat graphs with $W_{k}$ is given (up to a constant multiple) by evaluation of the cocycle $c_{\mathcal{F} a t}^{k}$ on the family.

Theorem 3.8 The cohomology class $\left[c_{\mathcal{F} a t}^{k}\right]$ is a multiple of the dual $\left[W_{k}\right]^{*}$ of the Witten cycle $W_{k}$. More precisely:

(1) The fat graph cocycle $c_{\mathcal{F} \text { at }}^{k}$ vanishes on any $2 k$-simplex of $\mathcal{F}$ at which is disjoint from $\mathcal{W}_{k}$.

(2) If $\Gamma, \Gamma^{\prime}$ are two objects of $\mathcal{W}_{k}$ of codimension $2 k$ (ie, having one vertex of valence $2 k+3$ and all other vertices trivalent). Then $\mathcal{F} a t / \Gamma \cong \mathcal{F} a t / \Gamma^{\prime}$ and $c_{\mathcal{F} \text { at }}^{k}$ takes equal values on corresponding $2 k$ simplices.

Proof For (1) we note that the cyclic set cocycle $c_{\mathcal{Z}}^{k}$ is applied to the angle sets of vertices. In order to be non-zero this set must increase in size $2 k$ times starting with 3 . The last vertex must have valence $\geq 2 k+3$ making the final fat graph an object of $\mathcal{W}_{k}$.

For (2) we note that any object $\Gamma^{\prime \prime}$ over $\Gamma$ differs from $\Gamma$ only at the vertex of valence $2 k+3$. So, there is a corresponding object over $\Gamma^{\prime}$ equal to $\Gamma^{\prime \prime}$ at this vertex and equal to $\Gamma^{\prime}$ away from the vertex. The cocycle $c_{\mathcal{Z}}^{k}$ is being applied only at this vertex. So, its value is the same on corresponding simplices. 
It remains to compute the value of the fat graph cocycle on the category $\mathcal{F} a t / \Gamma$. The realization of this category is the Stasheff associahedron (if we choose one object from each isomorphism class).

\subsection{The Stasheff associahedron}

It was Stasheff [19] who constructed the Stasheff associahedron $A_{n}$ (also called the Stasheff polyhedron) and showed that it was an $n-3$ disk. The reason it is called an associahedron is because it is an operad which continuously parametrizes the possible ways to multiply, say, $n-1$ loops in any loop space. This interpretation will not play a role in this paper.

For $n \geq 3$ let $\mathcal{A}_{n}$ be the poset of all isomorphism classes of planar trees with $n$ fixed leaves. This is a finite poset whose elements have various interpretations which we use interchangeably. One easy method to define this poset is to say that its elements are sets $\Gamma$ of unordered pairs $\{a, b\}$ of distinct integers modulo $n$ so that:

(1) $b-a \neq \equiv \pm 1 \bmod n$.

(2) Whenever $a<b<c<d<a+n$, the two pairs $\{a, c\}$ and $\{b, d\}$ are not both in the set $\Gamma$.

In that case $\Gamma$ represents the planar tree with vertices $1,2, \cdots, n$ in cyclic order so that the two arcs $(i, i+1)$ and $(j, j+1)$ bound two regions which come together at an internal edge if and only if $\{i, j\} \in \Gamma$. We will say that this edge separates the regions $i$ and $j$.

The set $\mathcal{A}_{n}$ is ordered by inclusion. It has a unique minimal element (the empty set in the above interpretation), so it is obviously contractible. Also, the height of any element is at most $n-3$. Thus the geometric realization $\left|\mathcal{A}_{n}\right|$ is $n-3$ dimensional.

We often view $\mathcal{A}_{n}$ as a category having a unique morphism $x \rightarrow y$ iff $x \geq y$.

Definition 3.9 The Stasheff associahedron $A_{n}$ is defined to be the geometric realization $\left|\mathcal{A}_{n}\right|$ of the category $\mathcal{A}_{n}$.

Now consider the case $n=2 k+3$. Let

$$
\Gamma_{0}>\Gamma_{1}>\cdots>\Gamma_{2 k}
$$

be a nondegenerate $2 k$-simplex in $\mathcal{A}_{2 k+3}$. Then $\Gamma_{0}$ is a trivalent graph (interior vertices are trivalent) with exactly $2 k$ internal edges. The internal edges can 
be numbered $e_{1}, e_{2}, \cdots, e_{2 k}$ so that the edge $e_{i}$ collapses in the $i^{\text {th }}$ step (from $\Gamma_{i-1}$ to $\left.\Gamma_{i}\right)$.

Now number the internal vertices. The first edge $e_{1}$ has two vertices which we label $v_{0}, v_{1}$ at random. For each other edge $e_{i}$ let $v_{i}$ be the endpoint of $e_{i}$ which is the furthest away from $v_{0}$. For $i \geq 2$ this is also the endpoint of $e_{i}$ furthest away from $v_{1}$ so the numbering of $v_{2}, \cdots, v_{2 k}$ remains unchanged if we switch $v_{0}, v_{1}$.

Let $a_{1}, a_{2}, a_{3}$ be, in cyclic order, the three regions which come together at the vertex $v_{0}$ so that $e_{1}$ separates the regions $a_{1}, a_{3}$. For $i=1,2, \cdots, 2 k$ let $b_{i}$ be the region which touches the vertex $v_{i}$ without touching the interior of the edge $e_{i}$.

Lemma $3.10 a_{1}, a_{2}, a_{3}, b_{1}, \cdots, b_{2 k}$ are distinct regions (distinct elements of the set $\{0,1,2, \cdots, 2 k+2\}$.

Proof For each region $r$ take the vertex in its boundary which is closest to the vertex $v_{0}$. This sends $b_{i}$ to $v_{i}$ and $a_{1}, a_{2}, a_{3}$ to $v_{0}$.

Definition 3.11 The orientation of the $2 k$-simplex $\Gamma_{0}>\Gamma_{1}>\cdots>\Gamma_{2 k}$ is defined to be the sign of the permutation $\left(a_{1}, a_{2}, a_{3}, b_{1}, \cdots, b_{2 k}\right)$ of the set $\{0,1, \cdots, 2 k+2\}$.

Note first that this orientation is well-defined. If we reverse (the names of) the vertices $v_{0}, v_{1}$ then $a_{1}, a_{2}, a_{3}, b_{1}$ become $a_{3}, b_{1}, a_{1}, a_{2}$ (an even permutation).

By Stasheff $A_{2 k+3}=\left|\mathcal{A}_{2 k+3}\right|$ is a $2 k$-disk. Consequently, the $2 k$-simplices can be oriented so that their homological boundary is the boundary sphere. We just need to check that we have one of the two consistent orientation conventions.

Proposition 3.12 Definition 3.11 gives a consistent orientation of the $2 k$ simplices of the Stasheff associahedron $A_{2 k+3}$.

Proof To check the consistency of our sign convention we only need to show that the sign changes under both of the following involutions:

(1) interchanging the numbering of two edges $e_{i}$ and $e_{j}$

(2) transforming the edge $e_{1}$ so that it goes the other way (separates $a_{2}, b_{1}$ instead of $\left.a_{1}, a_{3}\right)$. 
It is easy to see that both of these transformations changes the sign of the permutation.

The first involution merely switches the labels $b_{i}, b_{j}$ (when $i, j>1$ ). This is a transposition and thus odd. In the case $i=1$, we first consider that case when $e_{j}$ is (geometrically) adjacent to $e_{1}$. In that case we may assume that $v_{0}$ is the common vertex of $e_{1}, e_{j}$. Then when we switch $e_{1}, e_{j}$ the distance function to $v_{0}$ does not change so the vertices $v_{i}$ and the regions $b_{i}$ do not change for $i \neq 1, j$. The regions $b_{1}, b_{j}$ are interchanged and the regions $a_{1}, a_{2}, a_{3}$ are cyclically permuted. Consequently, the sign changes. All other permutations can be obtained by composing these two operations.

The second transformation changes only the regions $a_{1}, a_{2}, a_{3}, b_{1}$. They become $b_{1}, a_{1}, a_{2}, a_{3}$ (or $a_{2}, a_{3}, b_{1}, a_{1}$ ), an odd permutation.

Finally, we need to explain why it suffices to show that (1) and (2) switch the sign of the simplex.

Our $2 k$-simplex has $2 k+1$ faces. The last face does not count since it is on the boundary sphere. The $0^{\text {th }}$ face is given by

$$
\Gamma_{1}>\Gamma_{2}>\cdots>\Gamma_{2 k} .
$$

In $\Gamma_{1}$ the first edge $e_{1}$ is collapsed. This $2 k-1$ simplex is also the 0 -face of the $2 k$-simplex

$$
\Gamma_{0}^{\prime}>\Gamma_{1}>\cdots>\Gamma_{2 k}
$$

where $\Gamma_{0}^{\prime}$ is obtained from $\Gamma_{0}$ by operation (2) above. Consequently, we need this operation to switch the sign of the simplex so that the 0 -faces will homologically cancel.

The $i^{\text {th }}$ face of the $2 k$ simplex $\Gamma_{*}$ is given by deleting $\Gamma_{i}$ from the sequence. However, when $0<i<2 k$, this is the same as deleting the $i^{\text {th }}$ face of the $2 k$-simplex $\Gamma_{*}^{\prime}$ obtained from $\Gamma_{*}$ by switching the labels $e_{i}$ and $e_{i+1}$ (and their order of collapse). This is operations (1).

Consequently, the boundary of the $2 k$-chain given by the sum of all $2 k-$ simplices with signs as above is equal to the sum of their $2 k$-faces forming the boundary $2 k-1$ sphere.

\subsection{Computation of $c_{\mathcal{F} a t}^{k}\left(W_{k}\right)$}

We are now ready to compute the value of $c_{\mathcal{F} \text { at }}^{k}$ on the $2 k$-chain given by the sum of all simplices in $\mathcal{A}_{2 k+3}$ with signs according to our convention. First we 
note that $c_{\mathcal{F} a t}^{k}=0$ on many of these $2 k$ simplices. To have a chance to be non-zero we need the graphs $\Gamma_{i}$ in $\Gamma_{*}$ to have the property that they have one vertex of valence $i+3$ and all other internal vertices to be trivalent. Let $B_{k}$ be the $2 k$-chain consisting of only these $2 k$-simplices times appropriate signs:

$$
B_{k}=\sum \operatorname{sgn}\left(a_{1}, a_{2}, a_{3}, b_{1}, \cdots, b_{2 k}\right) \Gamma_{*}
$$

There are exactly

$$
\frac{(2 k+3) !}{6}
$$

permutations of the letters $a_{1}, a_{2}, a_{3}, b_{1}, \cdots, b_{2 k}$ which keep $a_{1}, a_{2}, a_{3}, b_{1}$ in cyclic order. These permutations corresponds to the $2 k$-simplex in the chain $B_{k}$ in a $2-1$ manner. In other words, $B_{k}$ has $(2 k+3) ! / 3$ terms.

To see this it helps to go backwards. Start at the terminal graph $\Gamma_{2 k}$. This has only one internal vertex. All regions converge at that vertex. If $b_{2 k}=j$ then the graph $\Gamma_{2 k-1}$ is obtained from $\Gamma_{2 k}$ by pulling region $j$ away from the center of the graph leaving a vertex at which all regions except for region $j=b_{2 k}$. To get $\Gamma_{i}$ from $\Gamma_{i+1}$ we pull away region $b_{i+1}$ from the central vertex. This applies even in the case $i=0$ but in that case the same result could have been obtained by "pulling away" the region labelled $a_{2}$. In other words, if we switch $a_{2}, b_{1}$ and we also switch $a_{1}, a_{3}$ then the resulting $2 k$ simplex is the same. There is a $2-1$ correspondence between permutations of the letters $a_{1}, a_{2}, a_{3}, b_{1}, \cdots, b_{2 k}$ which keep $a_{1}, a_{2}, a_{3}, b_{1}$ in cyclic order and $2 k$ simplices which occur in the $2 k$ chain $B_{k}$.

There is another way to look at this $2-1$ correspondence. The formula for the combinatorial Miller-Morita-Mumford class applies the cyclic set cocycle $c_{\mathcal{Z}}^{k}$ to exactly two vertices of the graph $\Gamma_{0}$ for each $2 k$-simplex $\Gamma_{*}$ which appears in $B_{k}$. These are the vertices $v_{0}, v_{1}$ of $\Gamma_{0}$ which merge in $\Gamma_{1}$. The permutations of the letters $a_{1}, a_{2}, a_{3}, b_{1}, \cdots, b_{2 k}$ which keep $a_{1}, a_{2}, a_{3}, b_{1}$ in cyclic order are in natural $1-1$ correspondence with the vertex-graph pairs upon which the cyclic set cocycles are to be evaluated.

Now we consider the situation. We have a vertex $v_{0}$ in a graph $\Gamma_{0}$. The angles at $v_{0}$ are labelled $a_{1}, a_{2}, a_{3}$. As we pass down to $\Gamma_{1}, \Gamma_{2}$, etc. the angles $b_{1}, b_{2}$, etc. are added. Consequently, the value of the cyclic set cocycle $c_{\mathcal{Z}}^{k}$ is given by:

$$
c_{\mathcal{Z}}^{k}\left(\Gamma_{*}, v_{0}\right)=\frac{(-1)^{k} k ! \sum_{i=1}^{3} \operatorname{sgn}\left(a_{i}, b_{1}, b_{2}, \cdots, b_{2 k}\right)}{(2 k) !(2 k+3) ! / 2}
$$

The value of the combinatorial cocycle $c_{\mathcal{F} \text { at }}^{k}$ on the $2 k$-chain $B_{k}$ is therefore given by:

$$
c_{\mathcal{F} a t}^{k}\left(B_{k}\right)=\frac{(2 k+3) !}{6} \frac{(-1)^{k} k ! E(X)}{(2 k) !(2 k+3) ! / 2}=\frac{(-1)^{k} k ! E(X)}{3(2 k) !}
$$


where $E(X)$ is the expected value of the random variable:

$$
X=\operatorname{sgn}\left(a_{1}, a_{2}, a_{3}, b_{1}, \cdots, b_{2 k}\right) \sum_{i=1}^{3} \operatorname{sgn}\left(a_{i}, b_{1}, b_{2}, \cdots, b_{2 k}\right)
$$

To compute $E(X)$ we note first that $X$ depends only on the relative positions of $a_{1}, a_{2}, a_{3}$. For example, if they are consecutive then $X=3$. More generally, if there are $p$ numbers between $a_{1}$ and $a_{2}$, ie,

$$
p= \begin{cases}a_{2}-a_{1}-1 & \text { if } a_{2}>a_{1} \\ a_{2}-a_{1}+2 k+2 & \text { otherwise }\end{cases}
$$

and $q$ numbers between $a_{2}$ and $a_{3}$, then

$$
\begin{aligned}
& \operatorname{sgn}\left(a_{1}, a_{2}, a_{3}, b_{1}, \cdots, b_{2 k}\right) \operatorname{sgn}\left(a_{1}, b_{1}, b_{2}, \cdots, b_{2 k}\right)=(-1)^{q} \\
& \operatorname{sgn}\left(a_{1}, a_{2}, a_{3}, b_{1}, \cdots, b_{2 k}\right) \operatorname{sgn}\left(a_{2}, b_{1}, b_{2}, \cdots, b_{2 k}\right)=(-1)^{p+q} \\
& \operatorname{sgn}\left(a_{1}, a_{2}, a_{3}, b_{1}, \cdots, b_{2 k}\right) \operatorname{sgn}\left(a_{3}, b_{1}, b_{2}, \cdots, b_{2 k}\right)=(-1)^{p}
\end{aligned}
$$

so

$$
X=(-1)^{p}+(-1)^{q}+(-1)^{p+q}=\left[1+(-1)^{p}\right]\left[1+(-1)^{q}\right]-1 .
$$

Let $Y$ be the random variable representing the number of spaces between $b_{1}$ and $a_{2}$ :

$$
Y= \begin{cases}a_{2}-b_{1}-1 & \text { if } a_{2}>b_{1} \\ a_{2}-b_{1}+2 k+2 & \text { otherwise }\end{cases}
$$

Then $Y$ takes values in the set $\{1,2, \cdots, 2 k\}$ with probabilities:

$$
P(Y=y)=\frac{y(2 k+1-y)}{\sum_{j=1}^{2 k} j(2 k+1-j)}
$$

We leave it as an exercise for the reader to show that value of the denominator is:

$$
\sum_{j=1}^{2 k} j(2 k+1-j)=\frac{1}{3} k(2 k+1)(2 k+2)
$$

(It suffices to verify this for $k=0,1,2,3$.)

Now we use the formula: $E(X)=\sum_{y=1}^{2 k} E(X \mid Y=y) P(Y=y)$. We compute the conditional expected value of $X+1$ :

$$
E(X+1 \mid Y=y)=\frac{1}{y(2 k-y+1)} \sum_{p=0}^{y-1} \sum_{q=0}^{2 k-y}\left[1+(-1)^{p}\right]\left[1+(-1)^{q}\right]
$$




$$
=\left(\frac{1}{y} \sum_{p=0}^{y-1}\left[1+(-1)^{p}\right]\right)\left(\frac{1}{2 k-y+1} \sum_{q=0}^{2 k-y}\left[1+(-1)^{q}\right]\right)
$$

If $y$ is odd then the right hand factor is 1 and the left hand factor is $1+\frac{1}{y}$. Similarly, if $y$ is even the left hand fact or is 1 and the right factor is $1+1 /(2 k-$ $y+1)$. Consequently,

$$
E(X+1 \mid Y=y)= \begin{cases}1+\frac{1}{y} & \text { if } y \text { is odd } \\ 1+\frac{1}{2 k-y+1} & \text { if } y \text { is even }\end{cases}
$$

Using the apparent symmetry between $y$ and $2 k-y+1$ we get:

$$
E(X)=2 \sum_{j=1}^{k} E(X \mid Y=2 j) P(Y=2 j)=\frac{2 \sum_{j=1}^{k} 2 j}{\sum_{j=1}^{2 k} j(2 k+1-j)}=\frac{3}{2 k+1}
$$

Plugging this into (18) we get:

$$
c_{\mathcal{F} a t}^{k}\left(B_{k}\right)=\frac{(-1)^{k} k ! E(X)}{3(2 k) !}=(-1)^{k} \frac{k !}{(2 k+1) !}
$$

This proves:

\section{Theorem 3.13}

$$
\left[c_{\mathcal{F} \text { at }}^{k}\right]=(-1)^{k} \frac{k !}{(2 k+1) !}\left[W_{k}\right]^{*}
$$

as elements of $H^{2 k}(\mathcal{F} a t ; \mathbb{Q}) \cong H^{2 k}\left(M_{g}^{s} ; \mathbb{Q}\right)$.

\subsection{Kontsevich cycles}

Suppose that $k_{1}, k_{2}, \cdots, k_{r}$ are positive integers. Then Kontsevich [10] defined the cycles

$$
W_{k_{1}, k_{2}, \cdots, k_{r}}
$$

in the space of fat graphs to be the set of all fat graphs having $r$ vertices $v_{1}, \cdots, v_{r}$ with valences $2 k_{1}+3,2 k_{2}+3, \cdots, 2 k_{r}+3$ respectively and no other vertices of valence $>3$. Kontsevich also conjectured in [10] that these were all related to the Miller-Morita-Mumford classes. Arbanello and Cornalba 1 made this more precise by conjecturing that their duals

$$
\left[W_{k_{1}, k_{2}, \cdots, k_{r}}\right]^{*} \in H^{\operatorname{deg}\left(k_{*}\right)}(\mathcal{F} a t ; \mathbb{Z})=H^{\operatorname{deg}\left(k_{*}\right)}\left(M_{g}^{s} ; \mathbb{Z}\right)
$$

should be polynomials in the adjusted Miller-Morita-Mumford classes $\widetilde{\kappa}_{j}$. Here the degree $\operatorname{deg}\left(k_{*}\right)$ of the weight $k_{*}=\left\{k_{1}, \cdots, k_{r}\right\}$ is given by

$$
\operatorname{deg}\left(k_{1}, \cdots, k_{r}\right)=2 k_{1}+\cdots+2 k_{r} .
$$


The weights are partially ordered by $\left\{j_{1}, \cdots, j_{s}\right\}<\left\{k_{1}, \cdots, k_{r}\right\}$ if $s<r$ and there is an epimorphism of sets $p:\{1, \cdots, r\} \rightarrow\{1, \cdots, s\}$ so that

$$
j_{t}=\sum_{i \in p^{-1}(t)} k_{i}
$$

Because of the easy to understand nature of the combinatorial classes $\left[c_{\mathcal{F} \text { at }}^{k}\right]$ we get the following corollary of the calculation in Theorem 3.13 .

Corollary 3.14 Consider the case when $k_{1}<k_{2}<\cdots<k_{r}$ occur with multiplicities $n_{1}, \cdots, n_{r}$. Then

$$
\left[c_{\mathcal{F} a t}^{k_{1}}\right]^{n_{1}} \cdots\left[c_{\mathcal{F} a t}^{k_{r}}\right]^{n_{r}}=\prod_{i=1}^{r} n_{i} !\left(\frac{(-1)^{k_{i}} k_{i} !}{\left(2 k_{i}+1\right) !}\right)^{n_{i}}\left[W_{k_{1}^{n_{1} \ldots k_{r}^{n}}}\right]^{*}+\text { lower terms }
$$

where the "lower terms" refers to rational linear combinations of dual Kontsevich classes $\left[W_{j_{1} \cdots j_{m}}\right]^{*}$ of weights $\left\{j_{1}, \cdots, j_{m}\right\}$ less than $\left\{\left(k_{1}\right)^{n_{1}}, \cdots,\left(k_{r}\right)^{n_{r}}\right\}$ in the partial ordering defined above.

Proof This is very elementary. Take the cup product of the fat graph cocycles

$$
c=c_{\mathcal{F} a t}^{p_{1}} \cup \cdots c_{\mathcal{F} \text { at }}^{p_{s}}
$$

where $s=\sum n_{i}$ and $p_{1}, \cdots, p_{s}$ are $k_{1}, \cdots, k_{r}$ with multiplicities $n_{1}, \cdots, n_{r}$.

When we evaluate the cocycle $c$ above on the $2 d$ simplex

$$
\Gamma_{*}=\left(\Gamma_{0} \rightarrow \cdots \Gamma_{2 d}\right)
$$

where $2 d=\operatorname{deg}\left(p_{*}\right)$ we will get zero unless there is a vertex of $\Gamma_{0}$ which increases in valence at each step until we reach $\Gamma_{2 p_{1}}$, then we need a vertex of $\Gamma_{2 p_{1}}$ which increases in valence until we reach $\Gamma_{2 p_{1}+2 p_{2}}$, etc. Assuming that $\Gamma_{2 d}$ has codimension $\leq 2 d$, this is possible if and only if the graph $\Gamma_{2 d}$ lies in a Kontsevich cycle $W_{j_{1}, \cdots, j_{m}}$ where

$$
\left\{j_{1}, \cdots, j_{m}\right\} \leq\left\{p_{1}, \cdots, p_{s}\right\}=\left\{\left(k_{1}\right)^{n_{1}}, \cdots,\left(k_{r}\right)^{n_{r}}\right\} .
$$

If $m=s$ and $j_{i}=p_{i}$ then the cocycle $c$ above, when evaluated on $\Gamma_{*}$ will be the product of coefficients

$$
\frac{(-1)^{k_{i}} k_{i} !}{\left(2 k_{i}+1\right) !}
$$

coming from each vertex of $\Gamma_{2 d}$ of valence $>3$ with a factor of $\prod n_{i}$ ! counting the number of permutations of these vertices which preserve their valences. 
Corollary 3.14 expresses monomials in the $\left[c_{\mathcal{F} a t}^{k}\right]$ 's as rational linear combinations of the Kontsevich cycles in such a way that the change of basis matrix is upper triangular with non-zero diagonal entries. The inverse of this matrix is upper triangular with diagonal entries inverse to those in Corollary 3.14. Thus we get the following.

\section{Corollary 3.15}

$$
\left[W_{k_{1}^{n_{1} \ldots k_{r}^{n}}}\right]^{*}=\prod_{i=1}^{r} \frac{1}{n_{i} !}\left(\frac{\left(2 k_{i}+1\right) !}{(-1)^{k_{i}} k_{i} !}\right)^{n_{i}}\left[c_{\mathcal{F} a t}^{k_{1}}\right]^{n_{1}} \cdots\left[c_{\mathcal{F} a t}^{k_{r}}\right]^{n_{r}}+\text { lower terms }
$$

where the "lower terms" are rational linear combinations of cup products of the form $\left[c_{\mathcal{F} \text { at }}^{j_{1}}\right] \cdots\left[c_{\mathcal{F} a t}^{j_{m}}\right]$ with weights $\left\{j_{1}, \cdots, j_{m}\right\}<\left\{\left(k_{1}\right)^{n_{1}}, \cdots,\left(k_{r}\right)^{n_{r}}\right\}$. In particular, these lower terms have smaller algebraic degree, ie, $m<\sum n_{i}$.

If we use the formula $\left[c_{\mathcal{F} a t}^{k}\right]=-2 \kappa_{k}$ proved in Theorem 5.1 below we obtain Corollary 0.2 in the introduction.

\section{Framed fat graphs}

In this section we will modify the arguments of the previous section to prove the Witten conjecture, namely, the adjusted Miller-Morita-Mumford classes $\widetilde{\kappa}_{k}$ are dual to $\left[W_{k}\right]$ :

$$
\widetilde{\kappa}_{k}=a_{k}\left[W_{k}\right]^{*} .
$$

The value of the coefficient $a_{k}$ is computed in the next section. In other words, we will show in this section that the analogue of Theorem 3.8 holds for $\widetilde{\kappa}_{k}$.

The idea is to replace fat graphs with framed fat graphs. By the Framed Graph Theorem [7] every graph has a framed structure unique up to contractible choice. Thus there is no obstruction to doing this. Framed fat graphs $(\Gamma, \phi)$, although more complicated than the underlying fat graphs $\Gamma$, have the advantage in that they determine framed functions on the surface $\Sigma_{\Gamma}$. Consequently, the adjusted Miller-Morita-Mumford class $\widetilde{\kappa}_{k}$ is given by evaluating the (extended) cyclic set cocycle $c_{\mathcal{Z}^{+}}^{k}$ on the 0 -cells of the framed structure.

By using a $D_{4 k+4}$-equivariant framing for all trees in the Stasheff associahedron $A_{2 k+2}$ we conclude that the extended cyclic set cocycle $c_{\mathcal{Z}^{+}}^{k}$ can only be nonzero in a neighborhood of the Witten cycle $W_{k}$, just as in the case of fat graphs. 


\subsection{The Framed Function Theorem}

Before we explain the Framed Graph Theorem we need to review the definition and statement of the Framed Function Theorem [5]. First we recall that a generalized Morse function (GMF) on a compact smooth manifold $M$ is a smooth function $f: M \rightarrow \mathbb{R}$ which has only Morse and birth-death singularities. Birth-death points are the unique codimension 1 singularities of smooth functions. They are points at which the function $f$ can be written as

$$
f(x)=x_{0}^{3}+\sum_{i \neq 0} \pm x_{i}^{2}+C
$$

with respect to some local coordinate system. Here $C$ is a constant and the number of negative signs in the sum $\sum \pm x_{i}^{2}$ is called the index of the birthdeath point.

A generic unfolding of a birth-death point is given by

$$
f_{t}(x)=x_{0}^{3}+t x_{0}+\sum_{i \neq 0} \pm x_{i}^{2}+C
$$

for $t$ in a small interval $(-\epsilon, \epsilon)$ about 0 . Note that when $t>0$ the function $f_{t}$ has no critical points (in the coordinate neighborhood). For $t<0$ the function $f_{t}$ has two critical points in "cancelling position."

Definition 4.1 A framed function on a smooth manifold $M$ is a GMF $f: M \rightarrow$ $\mathbb{R}$ together with a tangential framing of the nonpositive eigenspace of the second derivative $D^{2} f$ at each critical point so that the last framing vector points in the positive cubic direction $\left(\frac{\partial}{\partial x_{0}}\right)$ at every birth-death point. In a family of framed functions we assume that the framing vectors vary continuously.

Theorem 4.2 [5] The space of framed functions on $M$ is $\operatorname{dim} M-1$ connected.

This theorem is not quite good enough in the present situation. We need to know that the space of framed functions on a compact surface is contractible. We cannot prove this but we have another theorem which is just as good for our purposes: the Framed Graph Theorem which implies that compact surfaces admit canonical framed functions which are unique up to contractible choice. These framed functions have the property that they have critical points only in indices 0,1 . 


\subsection{The Framed Graph Theorem}

We review the concept of a framed graph. It helps to keep in mind that this is the combinatorial structure associated with a framed function. These framed functions will have only three kinds of singularities:

(1) Morse points of index 0 (local minima).

(2) Morse points of index 1 (maxima in dim 1 and saddle points in dim 2).

(3) Birth-death points of index 0 (where the above two cancel).

We note that the framing of the framed function gives an orientation of the index 1 Morse points resulting in an oriented 1-dimensional cell complex as the core of our manifold (the domain of the framed function).

Definition 4.3 A framed graph is an oriented 1-dimensional cell complex $X$ in which 1-cells are allowed to be attached on the interiors of other 1-cells in some partial ordering of the set of cells and which have a designated collection of collapsing pairs. Collapsing pairs consist of a 1-cell and target 0-cell.

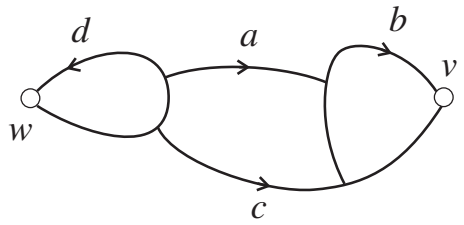

Figure 1

To expand further on our analogy with framed functions we note that in a 1-parameter family of framed functions, the cells corresponding to the critical points will slide over each other discontinuously. In the combinatorial setting we need to do this continuously. Thus the 1 -cells need to be allowed to attach to the interiors of other 1-cells. For functions we note that the critical points are ordered according to critical value and that cells can only slide over cells lower in this ordering. Also a birth-death point can be interpreted as a pair of cancelling critical points with the same critical value. Thus the partial ordering of the cells in our framed graph should make collapsing pairs equivalent in the partial ordering so that, eg, if $(e, v)$ forms a collapsing pair and $e^{\prime}$ is another 1 -cell, if $e^{\prime}$ attaches to $v$ then $e$ cannot be attached to the interior of $e^{\prime}$ (since $e^{\prime}<e \sim v<e^{\prime}$ would be a contradiction). Thus in Figure 1 neither $(b, v)$ nor $(d, w)$ is allowed to be a collapsing pair. 
In order to understand the morphisms between framed graphs we need to look at the underlying graph $\Gamma$ of a framed graph $X$. This graph will have vertices of valence 2 corresponding to 0 -cells which occur in the centers of edges. The leaves (vertices of valence 1) are also necessarily 0 -cells. However, vertices of valence $\geq 3$ may or may not be 0 -cells.

If a vertex of the underlying graph $\Gamma$ is not a 0 -cell then it must lie on the interior of a 1-cell. Consequently, two of the incident half-edges must form part of a 1-cell and the other incident half-edges must be ends of other 1-cells. (A 1-cell cannot be attached to the interior of itself by the partial ordering rule and a 1 -cell in a cancelling pair cannot have source equal to its target 0 -cell by the same rule.)

Strictly speaking, morphisms of framed graphs will be graph epimorphisms as in the category of fat graphs. However, we want to interpret them as handle slides. For example, if a 1-cell $a$ attached to the center of another 1 -cell $b$ then by collapsing one or the other half of $b$ we obtain what looks like a sliding of the attaching map of $a$ to one or the other endpoint of $b$. Thus a collapse of a portion of a 1-cell can be viewed as a handle slide.

Collapsing pairs are allowed to collapse to their source. They are also allowed to "unpair." This is analogous to the possible deformations of a birth-death point of index 0 . It can either resolve into a nonsingular function (collapsing the cell pair) or it can resolve into a pair of distinct Morse points. The latter possibility we represent as "unpairing" the cancelling pair, ie, keeping the pair but removing their designation (demoting them).

To emphasize the fact that we consider a framed graph $X$ as being an embellishment of the underlying combinatorial graph $\Gamma$ we write $X=(\Gamma, \phi)$ where $\phi$ represents the framed structure of $\Gamma$ given by $X$.

Definition 4.4 A morphism of framed graphs $\left(\Gamma_{0}, \phi\right) \rightarrow\left(\Gamma_{1}, \psi\right)$ is a graph epimorphism $f: \Gamma_{0} \rightarrow \Gamma_{1}$ with the property that:

(1) The collapsing trees $f^{-1}(v)$ do not contain any entire 1-cells unless they form part of a collapsing pair in $\Gamma_{0}$.

(2) Every 1-cell of $\Gamma_{1}$ is the image of a 1 -cell of $\Gamma_{0}$ with the induced orientation.

(3) Every 0 -cell of $\Gamma_{1}$ is the image of a 0 -cell of $\Gamma_{0}$.

(4) Every collapsing pair of $\Gamma_{1}$ is the image of a collapsing pair of $\Gamma_{0}$. 
We leave it to the reader to show that morphisms are closed under composition. Also we note that condition (2) implies condition (3). Another very useful observation is that $\Gamma_{0} \simeq \Gamma_{1}$ and therefore they have the same Euler characteristic. A certain number of collapsing pairs will disappear according to (1), but, in order to preserve the Euler characteristic, the remaining 0 -cells of $\Gamma_{0}$ must map to distinct 0 -cells of $\Gamma_{1}$. This implies the following.

Proposition 4.5 Under a morphism $f:\left(\Gamma_{0}, \phi\right) \rightarrow\left(\Gamma_{1}, \psi\right)$ each collapsing pair $(e, v)$ of $\Gamma_{0}$ will be transformed in one of three ways:

(1) $(e, v)$ will collapse to the source of e. (This removes one 0 -cell and one 1 -cell from the framed graph.)

(2) $(e, v)$ maps to a collapsing pair $(f(e), f(v))$ in $\Gamma_{1}$.

(3) $(e, v)$ is unpaired, ie, $f(e)$ is a 1 -cell and $f(v)$ is a 0 -cell but $f(e), f(v)$ do not form a collapsing pair.

For the purpose of giving the definition of a morphism (4.4) we allowed our underlying graph to have vertices of valence 2 . Normally this is not useful. We define the reduced graph of a framed graph to be the underlying graph with the valence 2 vertices "smoothed" in the sense that the two incident half edges are joined together. This causes a problem only if the graph is a circle. We regard the placement of the 0 -cells of valence 2 to be part of the framed structure $\phi$ of the reduced graph.

The Framed Graph Theorem says that any family of graphs admits a family of framed structures and that this structure can be specified on any closed subcomplex of the parameter space. (Consequently, the framed structure is unique up to framed homotopy.) However, in this paper we have not defined the notion of "family of graphs" for arbitrary graphs. We have however talked about families of fat graphs.

\subsection{Framed fat graphs}

A framed fat graph is simply a fat graph with a framed structure. Given a morphism of framed graphs $\left(\Gamma_{0}, \phi\right) \rightarrow\left(\Gamma_{1}, \psi\right)$ and a fat graph structure on $\Gamma_{0}$ there is an induced fat graph structure on $\Gamma_{1}$ since every tree in a subdivided $\Gamma_{0}$ induces a cyclic ordering of incident edges. A morphism of framed fat graphs is a morphism of framed graphs with compatible fat graph structures.

The Framed Graph Theorem implies that any family of fat graphs (ie, continuous mapping into the geometric realization $|\mathcal{F} a t|)$ admits a continuous family of 
framed structures which can be specified on any subcomplex of the parameter space.

The main theorem is that the adjusted Miller-Morita-Mumford class $\widetilde{\kappa}_{k}$ is given by applying the extended cyclic set cocycle $c_{\mathcal{Z}^{+}}^{k}$ to the 0 -cells of the framed structure. This follows from an examination of the framed functions on the punctured surfaces $\Sigma_{\Gamma}$ which are associated to the framed fat graphs.

\subsection{Relation to Morse theory}

Suppose that $\Sigma \rightarrow E \stackrel{p}{\rightarrow} M$ is a smooth oriented surface bundle with a fiberwise Riemannian metric. In case $M$ has a boundary $\partial M$, we assume that $E \mid \partial M=$ $p^{-1}(\partial M)$ is a trivial bundle $E \mid \partial M \cong \partial M \times \Sigma$. Let $\partial_{v} E$ be the subbundle of $E$ with fiber $\partial \Sigma$ (so that $\partial E=\partial_{v} E \cup E \mid \partial M$ ). Since $\partial \Sigma$ is a disjoint union of circles the mapping $\partial_{v} E \rightarrow M$ is the composition of an oriented circle bundle $\partial_{v} E \rightarrow \widetilde{M}$ with a covering map $\pi: \widetilde{M} \rightarrow M$. We recall the definition of the Miller-Morita-Mumford class. Take the Euler class $e_{E} \in H^{2}\left(E, \partial_{v} E ; \mathbb{Z}\right)$ of the vertical tangent bundle of $E$. Then the adjusted Miller-Morita-Mumford class is given by $\widetilde{\kappa}_{k}=p_{*} e_{E}^{k+1} \in H^{2 k}(M, \partial M ; \mathbb{Z})$ where

$$
p_{*}: H^{*}(E, \partial E ; \mathbb{Z}) \rightarrow H^{*-2}(M, \partial M ; \mathbb{Z})
$$

is the push-down operator. See [15] for more details. The usual Miller-MoritaMumford class $\kappa_{k} \in H^{2 k}(M, \partial M ; \mathbb{Z})$ is related to $\widetilde{\kappa}_{k}$ by

$$
\kappa_{k}=\widetilde{\kappa}_{k}+\pi_{*}\left(\gamma^{k}\right)
$$

where $\gamma \in H^{2}(\widetilde{M}, \partial \widetilde{M} ; \mathbb{Z})$ is the Euler class of the oriented circle bundle $\partial_{v} E \rightarrow$ $\widetilde{M}$ and $\pi_{*}: H^{*}(\widetilde{M}, \partial \widetilde{M} ; \mathbb{Z}) \rightarrow H^{*}(M, \partial M ; \mathbb{Z})$ is the push-down map.

Let $f: E \rightarrow \mathbb{R}$ be any generic smooth function whose fiberwise gradient $\nabla f_{t}$ points outward along the boundary $\partial \Sigma_{t}$ of every fiber $\Sigma_{t}$. Since $E \mid \partial M \cong$ $\Sigma \times \partial M$ we may assume that $f_{t}: \Sigma_{t}=\Sigma \rightarrow \mathbb{R}$ is a fixed Morse function for all $t \in \partial M$.

Since $f$ is generic, its vertical singular set $\Sigma(f)$ is a codimension 2 submanifold of $E$ which is disjoint from $\partial_{v} E$ and which is a product bundle over $\partial M$. Since $\Sigma(f)$ is the inverse image under the vertical gradient $\nabla f_{t}$ of the zero section of the vertical tangent bundle of $E$ we have the following well-known observation.

Proposition 4.6 The vertical singular set $\Sigma(f)$ is Poincaré dual to the Euler class $e_{E}$ of the vertical tangent bundle of $E$. 
Remark 4.7 This is not quite correctly stated since $\Sigma(f)$ meets the boundary of $E$. What we mean is that cup product with the Euler class $e_{E}$ is equivalent to restriction to $\Sigma(f)$. In particular, the $k+1$ st power $e_{E}^{k+1}$ is equivalent to the restriction to $\Sigma(f)$ of $e_{E}^{k}$ in the sense that they have the same push-down in $H^{2 k}(M, \partial M ; \mathbb{R})$.

Now suppose that $f: E \rightarrow \mathbb{R}$ is a fiberwise framed function which is still fixed over $\partial M$. The framed structure gives a trivialization of the vertical tangent bundle along birth-death points and saddle points (and maxima if there are any). Also we can choose a trivialization over $\partial M$. This gives the following.

Corollary 4.8 The adjusted Miller-Morita-Mumford classes $\widetilde{\kappa}_{k}$ are given by pushing down the restriction to the index 0 singular set $\Sigma^{0}(f)$ of the $k^{\text {th }}$ power $e_{E}^{k}$ of the vertical Euler class $e_{E}$ :

$$
\widetilde{\kappa}_{k}=p_{*}\left(e_{E}^{k} \mid \Sigma^{0}(f)\right)
$$

On the category of framed fat graphs we need to use the extended cyclic set cocycle $c_{\mathcal{Z}^{+}}^{k}$ on the set of 0 -cells. This takes into account the trivialization of the vertical tangent bundle at the places where the 0 -cell is part of a collapsing pair.

Definition 4.9 Suppose that $\Gamma_{*}=\left(\Gamma_{0} \rightarrow \Gamma_{1} \rightarrow \cdots \rightarrow \Gamma_{2 k}\right)$ is a $2 k$-simplex in the category of framed fat graphs. Then let $z^{k}\left(\Gamma_{*}\right)$ be given by

$$
z^{k}\left(\Gamma_{*}\right)=\sum c_{\mathcal{Z}^{+}}^{k}\left(C(v) \rightarrow C\left(f_{1}(v)\right) \rightarrow \cdots \rightarrow C\left(f_{2 k}(v)\right)\right)
$$

where the sum is over all 0 -cells $v$ of $\Gamma_{0}$ which survive (do not collapse) in $\Gamma_{2 k}$. We call this the $0-$ cell cocycle.

Since a framed structure on a fat graph $\Gamma$ gives a framed function on the punctured surface $\Sigma_{\Gamma}$ (See the last section of [7]), Corollary 4.8 gives the following.

Corollary 4.10 The adjusted Miller-Morita-Mumford classes $\widetilde{\kappa}_{k}$ are given by the 0-cell cocycle applied to the transverse families of framed fat graphs associated to the surface bundle $\Sigma \rightarrow E \rightarrow M$.

By transverse we mean that the underlying family of fat graphs satisfies Proposition 3.7 . 


\subsection{Localizing to Witten cycles}

We would like to show that the only terms in $z^{k}\left(\Gamma_{*}\right)$ which can be non-zero are the ones in which the last vertex $f_{2 k}(v)$ has valence $2 k+3$, ie, $\Gamma_{2 k}$ is in the Witten cycle $W_{k}$. This is very close to being true.

There are two ways that this might fail. The first is that framed fat graphs have 0 -cells of valence 2 . The second is that the extended cyclic set cocycle $c_{\mathcal{Z}}^{k}=$ can be non-zero even if the last two sets have the same size. Thus the worst case is:

$$
|C(v)|=2,\left|C\left(f_{1}(v)\right)\right|=3, \cdots,\left|C\left(f_{2 k-1}(v)\right)\right|=2 k+1=\left|C\left(f_{2 k}(v)\right)\right|
$$

where $v, f_{1}(v), \cdots, f_{2 k-1}(v)$ are paired $0-$ cells and $f_{2 k}(v)$ is unpaired.

In order to prevent this we will show that the framed structure on a transverse $2 k$-parameter family of fat graphs can be chosen so that all "massive" points are unpaired. By a massive point we mean a 0 -cell of valence $\geq k+3$ (codim $\geq k)$.

If $k \geq 2$ then $2 k+1 \geq k+3$ so the worse case (22) will not occur if all massive points are unpaired. The case $k=1$ is treated separately.

Assume for a moment that $k \geq 2$ and all massive points are unpaired. Then the vertex $f_{2 k-1}(v)$ must be unpaired and the worst case (22) does not occur. Instead the worst case is

$$
|C(v)|=2,\left|C\left(f_{1}(v)\right)\right|=3, \cdots,\left|C\left(f_{2 k}(v)\right)\right|=2 k+2
$$

with all 0 -cells $f_{i}(v)$ unpaired. However, this is not possible since the associahedron $A_{2 k+2}$ giving the unfolding of a vertex of valence $2 k+2$ is a $2 k-1$ disk which forces the $2 k$-simplex (23) to be degenerate. To make this argument valid we need to choose a $D_{4 k+4}$-equivariant framing of the trees in the Stasheff associahedron $A_{2 k+2}$. We will give an explicit such framing which is compatible with our first condition, (ie, massive points are unpaired).

Of course, any triangulation of a $2 k-1$ disk will have no nondegenerate $2 k-$ simplices. The essential point is the dihedral $\left(D_{4 k+4}\right)$ symmetry of the framing which allows us to give a product structure to the framed structures in a neighborhood of the arcs of $W_{k-\frac{1}{2}}$ graphs (having a $2 k+2$ valent vertex) in our generic $2 k$-parameter family of fat graphs. The final result will be that the 0 -cell cocycle $z^{k}$ will only be non-zero at two places:

(1) The final vertex $f_{2 k}(v)$ has valence $2 k+3$ forcing the final fat graph $\Gamma_{2 k}$ to lie in the Witten cycle $W_{k}$. 
(2) The final vertex has valence $2 k+2$ and lies near the boundary of the associahedron $A_{2 k+3}$ where the dihedral symmetry of the lower associahedron $A_{2 k+2}$ is broken.

This is explained in detail in the last section.

\subsection{Massive points}

The first step is to arrange for all massive vertices to be unpaired. To do this we need the following relative version of the Framed Graph Theorem. Fortunately, it follows from the absolute version.

Let $\Gamma: \mathcal{P} \rightarrow \mathcal{F}$ at be a functor from any small category $\mathcal{P}$ into the category of connected fat graphs. Then we get an induced continuous family of fat graphs $\Gamma(t), t \in|\mathcal{P}|$. Suppose that each $\Gamma(X), X \in \mathcal{P}$ has two disjoint subgraphs $A(X)$ and $B(X)$ which contain all the vertices of $\Gamma(X)$. Thus

$$
E(X)=\Gamma(X)-(A(X) \cup B(X))
$$

is a disjoint union of open edges. Suppose that this decomposition is natural in the sense that every morphism $X \rightarrow Y$ in $\mathcal{P}$ sends $A(X)$ to $A(Y), B(X)$ to $B(Y)$ and $E(X)$ to $E(Y)$. Then we get continuous families of subgraphs $A(t), B(t) \subseteq \Gamma(t)$ for $t \in|\mathcal{P}|$ and the number of elements of $E(t)$ is locally constant on $|\mathcal{P}|$.

More generally, suppose that $K$ is a closed subcomplex of (a subdivision of) $|\mathcal{P}|$. For each $t \in K$ suppose we have subgraphs $A(t), B(t)$ of $\Gamma(t)$ containing all the vertices of $\Gamma(t)$ so that

$$
E(t)=\Gamma(t)-(A(t) \cup B(t))
$$

is a disjoint union of open edges which vary continuously with $t \in K$ and the number of which is locally constant over $K$.

Theorem 4.11 Let $A(t), B(t) \subseteq \Gamma(t), t \in K$, be as described above. Then any continuous family of framed structures on the subgraphs $A(t), t \in K$, extends to a continuous family of framed structures on $\Gamma(t)$ with the property that:

(1) $B(t)$ is a subgraph of $\Gamma(t)$ for all $t \in K$.

(2) None of the 1-cells in $E(t)$ forms a collapsing pair with a vertex in $A(t) \cup B(t)$. 
Furthermore, the relative version of this statement is true: The framed structure on $\Gamma(t)$ extending the given framed structure on $A(t)$ can be specified for $t \in L$ for any subcomplex $L$ of $K$ provided that the above two conditions hold over $L$.

Proof The absolute version is easy. Using the Framed Graph Theorem we can simply choose a continuous framed structure on $B(t)$ for all $t \in K$ and then take the following framed structure on the edges of $E(t)$ :

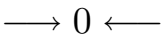

(Place a $0-$-cell in the center and form two 1-cells oriented towards the center.)

For the relative case we use the relative version of the Framed Graph Theorem to construct a continuous family of framed structures on $B(t)$ extending the given structure over $L$. Then we use the fact that the space of framed structures on an interval with specified boundary behavior is contractible (the case $\mathcal{F} \mathcal{G}_{11}(I)$ of Theorem 8.3.2 of [7]).

Now let $\mathcal{P}_{2 k}$ denote the full subcategory of $\mathcal{F}$ at consisting of fat graphs of codimension $\leq 2 k$ with at least one massive vertex, ie, with valence $\geq k+3$. We want to construct subgraphs $A(t)$ of $\Gamma(t)$ for each $t \in\left|\mathcal{P}_{2 k}\right|$ containing the massive vertices of $\Gamma(t)$ and we want to use Theorem 4.11 above to specify the framed structure on $A(t)$ (so that the massive vertex is not a paired 0-cell).

Consider a $p$-simplex $\Gamma_{0} \rightarrow \cdots \rightarrow \Gamma_{p}$ in $\mathcal{P}_{2 k}$. If $\Gamma_{0}$ has two massive vertices then it has codimension $2 k$ and $\Gamma_{i} \cong \Gamma_{0}$ for all $i$. So suppose that $\Gamma_{0}$ has a unique massive vertex $v$. Let $f_{i}(v) \in \Gamma_{i}$ be the image of $v$.

Let $T_{i} \subseteq \Gamma_{i}$ be the inverse image of $f_{p}(v) \in \Gamma_{p}$. Then $T_{i}$ determines a continuous family of subgraphs $T(t) \subseteq \Gamma(t)$ for all $t \in \Delta^{p}$. However, this is not a suitable choice for $A(t)$ since these are graphs having vertices connected to a massive vertex by two edges.

Our second attempt is to define $A(t)$ to be the subgraph of $\Gamma(t)$ consisting of the massive vertex and any connected "short edges" where by a short edge we mean an edge of length $\leq \lambda$ for some fixed $\lambda \approx \frac{1}{2}$ and by connected we mean either directly or through other short edges. (Thus $A(t)$ is connected with all edges of length $\leq \lambda$.) However, there is one problem with this definition. There might be edges of length slightly larger than $\lambda$ whose length decreases to $\Lambda$. We will avoid this and other unseen problems with a more formal approach.

First, choose a small positive real number $\epsilon$. $\left(0<\epsilon<\frac{1}{2 k+3}\right.$ is sufficient.) Then, for each $k \leq j \leq 2 k$ and $t \in\left|\mathcal{P}_{2 k}\right|$ we define: 
(1) The closed $j$-weight of $\Gamma(t)$ to be the codimension of the massive point of $\Gamma(t)$ plus the number of edges of length $\leq j \epsilon+\frac{1}{2}$ (call them $j$-short edges) which are connected to the massive point either directly or through other $j$-short edges.

(2) The open $j$-weight of $\Gamma(t)$ is defined to be the codimension of the massive point plus the number of edges of length strictly less than $j \epsilon+\frac{1}{2}$ (call them strictly $j$-short edges) which are connected to the massive point by strictly $j$-short edges.

Note that closed $j$-weight is upper semicontinous (being bounded above is an open condition) and open $j$-weight is lower semicontinuous (being bounded below is open). Also

$$
\text { open } j \text {-weight } \leq \text { closed } j \text {-weight. }
$$

For each $0 \leq j \leq k$ let $K_{j}$ be the set of all $t \in\left|\mathcal{P}_{2 k}\right|$ so that the closed $j$-weight of $\Gamma(t)$ is $\geq k+j$ and equal to the open $(j+1)$-weight of $\Gamma(t)$. This is a closed condition since it can be written as:

$$
\text { open }(j+1) \text {-weight }(\Gamma(t))-\operatorname{closed} j \text {-weight }(\Gamma(t)) \leq 0
$$

Lemma 4.12 $\left|\mathcal{P}_{2 k}\right|$ is a union of the closed subcomplexes $K_{j}$ for $0 \leq j \leq k$.

Proof For any $t \in\left|\mathcal{P}_{2 k}\right|$ let $\lambda_{t}: I \rightarrow \mathbb{N}$ be defined as follows. $\lambda_{t}(x)$ is equal to the codimension of the massive point of $\Gamma(t)$ plus the number of attached short edges where short means of length $\leq x$. Then $K_{j}$ is the set of all $t \in$ $\left|\mathcal{P}_{2 k}\right|$ so that $\lambda_{t}\left(j \epsilon+\frac{1}{2}\right) \geq k+j$ and $\lambda_{t}$ is constant on the half-open interval $\left[j \epsilon+\frac{1}{2},(j+1) \epsilon+\frac{1}{2}\right)$.

For any $t \in\left|\mathcal{P}_{2 k}\right|$ we note that $\lambda_{t}\left(\frac{1}{2}\right) \geq k$. Consequently, there exists a $j \geq 0$ so that $\lambda_{t}\left(j \epsilon+\frac{1}{2}\right) \geq k+j$. Choose the largest such $j \leq k$. If $t \notin K_{j}$ then we must have $\lambda_{t}(x)>\lambda_{t}\left(j \epsilon+\frac{1}{2}\right)$ for some $x<(j+1) \epsilon+\frac{1}{2}$. This implies that $\lambda_{t}\left((j+1) \epsilon+\frac{1}{2}\right) \geq k+j+1$ contradicting the maximality of $j$.

For each $t \in K_{j}$, let $A_{j}(t) \subseteq \Gamma(t)$ be the union of the massive points and all connected $j$-short edges (of length $\leq j \epsilon+\frac{1}{2}$ ). Let $E_{j}(t)$ be the set of edges connected to $A_{j}(t)$. By definition of $K_{j}$, each edge in $E_{j}(t)$ has length $\geq(j+1) \epsilon+\frac{1}{2}$. Consequently, the number of edges in $E_{j}(t)$ is a locally constant function of $t \in K_{j}$ so the conditions of Theorem 4.11 are satisfied.

Now we can specify the framing of $A_{j}(t)$. We take the 0 -cells to be the vertices and the 1-cells to be the edges oriented away from the massive point. We 
designate a 1-cell and its target vertex to be a collapsing pair if and only if its length is $\leq \frac{1}{4}$. We call this framing the radial framing of $A_{j}(t)$ centered at the massive point.

We extend this framing to the rest of $\Gamma(t)$ using Theorem 4.11 by downward induction on $j$. To check the relative condition note that if $t \in K_{\ell}$ for $\ell>j$ then $A_{j}(t) \subseteq A_{\ell}(t)$ so $A_{j}(t)$ is already radially framed. (And any edges of $A_{\ell}(t)$ which are not in $A_{j}(t)$ have length $>\frac{1}{2}$ so are not paired.) By the framed graph theorem this framing can also be extended to all fat graphs of codimension $\leq 2 k$. This shows:

Lemma 4.13 Framed structures can be chosen for all fat graphs of codimension $\leq 2 k$ so that all massive points are unpaired.

\subsection{The case $k=1$}

Now consider the special case $k=1$. The argument using massive points fails in this case. However, there is a simple reason that the desired statement still holds, ie, the 0 -cell cocycle $z^{1}\left(\Gamma_{*}\right)$ is still zero when $f_{1}(v)$ is a paired 0 -cell. The reason is that, when $v$ is a paired 0 -cell of valence 2 and $f_{1}(v)$ is a paired 0 -cell of valence 3 , there is only one possible geometry as shown in Figure 2 If $f_{2}(v)$ is unpaired with valence 3 then:

$$
c_{\mathcal{Z}^{+}}^{1}\left(C(v) \rightarrow C\left(f_{1}(v)\right) \rightarrow C\left(f_{2}(v)\right)\right)=-s_{1}\left(C(v) \rightarrow C\left(f_{1}(v)\right)\right)=\frac{\operatorname{sgn}(a b a)}{2 ! \cdot 2 \cdot 3}=0
$$

Therefore:

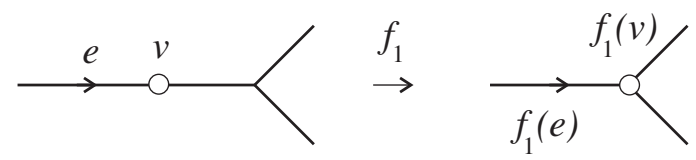

Figure 2: Unique geometry allowing $f_{1}(v)$ to be paired with valence 3

Lemma 4.14 For all $k \geq 1$ there are no non-zero terms in the 0 -cell cocycle unless the last vertex $f_{2 k}(v)$ has codimension $\geq 2 k-1$ (assuming that massive points are unpaired).

\subsection{Equivariant framing of the Stasheff associahedron $A_{2 k+2}$}

An equivariant framing of the Stasheff associahedron $A_{n}$ for any $n$ is easy to describe. We simply take the radial framing centered at the center of mass of each object of $\mathcal{A}_{n}$ (Definition [3.9). 
Definition 4.15 Suppose that $\Gamma$ is a tree with $n$ leaves and no bivalent vertices (equivalent to an object of $\mathcal{A}_{n}$ ). Then the center of mass of $\Gamma$ is defined to be either:

(1) The midpoint of the unique edge $e$ of $\Gamma$ with the property that each endpoint of $e$ is connected to half the leaves of $\Gamma$ by paths disjoint from the interior of $e$ (Figure 3 ).

(2) If no such edge exists then the unique vertex $v$ of $\Gamma$ having the property that no component of $\Gamma-e$ has more than half the leaves of $\Gamma$.

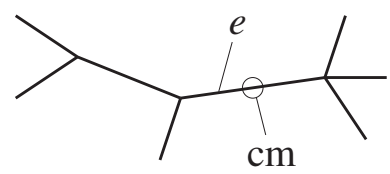

Figure 3: Center of mass (circle) for tree with 6 leaves

We leave it to the reader to verify that the center of mass is well-defined. Note that case (1) is possible only if $n$ is even.

Proposition 4.16 Let $n=2 k+2$ or $2 k+3$.

(1) Any morphism $\Gamma_{1} \rightarrow \Gamma_{2}$ in $\mathcal{A}_{n}$ sends the center of mass of $\Gamma_{1}$ to the center of mass of $\Gamma_{2}$.

(2) If $\Gamma \in \mathcal{A}_{n}$ has a massive vertex $v$ then $v$ is the center of mass.

In a $n-1$ parameter family of fat graphs, a fat graph $\Gamma$ could be a member of two Stasheff associahedra $A_{n}$. Thus, in order to have a well-defined framing, we will subdivide the associahedron making it into a union of a collar neighborhood of the boundary and a smaller half-sized copy of the associahedron which we call $A_{n}^{1 / 2}$. (See Figure 4 )

More precisely, $A_{n}^{1 / 2} \subseteq A_{n}=\left|\mathcal{A}_{n}\right|$ is defined to be the set of all

$$
\left(t, \Gamma_{*}\right) \in \Delta^{p} \times\left\{\Gamma_{0} \rightarrow \cdots \rightarrow \Gamma_{p}\right\}
$$

with the properties:

(a) The last tree $\Gamma_{p}$ contains a vertex of valence $n$ and

(b) $t_{p} \geq \frac{1}{2}$ (1 minus the superscript of $A_{n}$ ). 


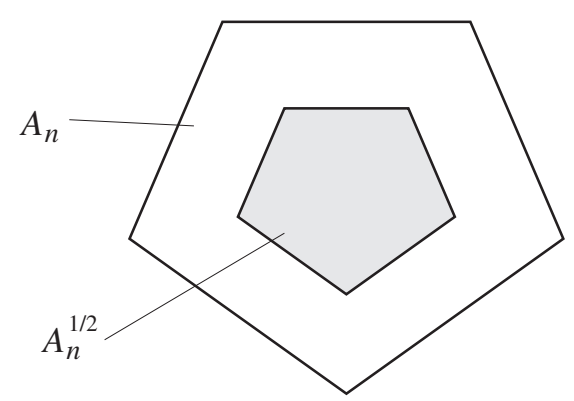

Figure 4: The small copy $A_{n}^{1 / 2}$ does not touch any other $A_{n}$.

Note: An edge of $\Gamma_{i}$ that collapses in $\Gamma_{i+1}$ has length $t_{0}+\cdots+t_{i}$ in $\Gamma(t)$. Thus $t_{p} \geq \frac{1}{2}$ is equivalent to saying that all edges which collapse in $\Gamma_{p}$ have length $\leq \frac{1}{2}$ in $\Gamma(t)$.

Now we restrict to the case $n=2 k+2$. The plan is to take any $2 k$ parameter family of fat graphs, subdivide any associahedron $A_{2 k+2}$ which occurs and take the radial framing of the half-sized subcomplex $A_{n}^{1 / 2}$ about the center of mass. It should be obvious that no fat graph lies in more than one such subcomplex.

We go through the details. Let $\mathcal{C}_{2 k}$ denote the full subcategory of $\mathcal{F} a t$ consisting of fat graphs of codimension $\leq 2 k$ which do not lie in the Witten cycle $W_{k}$, ie, do not contain vertices of valence $2 k+3$. Let $\Gamma(t), t \in\left|\mathcal{C}_{2 k}\right|$ be the corresponding family of metric fat graphs.

Let $P_{2 k+2}^{1 / 2}$ denote the subset of $\left|\mathcal{C}_{2 k}\right|$ corresponding to $A_{2 k+2}^{1 / 2}$, ie, the set of all

$$
\left(t, \Gamma_{*}\right) \in \Delta^{p} \times\left\{\Gamma_{0} \rightarrow \cdots \rightarrow \Gamma_{p}\right\} \subseteq\left|\mathcal{C}_{2 k}\right|
$$

with the properties:

(a) $\Gamma_{p}$ contains a vertex of valence $2 k+2$ and

(b) $t_{p} \geq \frac{1}{2}$.

For each $t \in P_{2 k+2}^{1 / 2}$ let $A(t)$ be the tree in $\Gamma(t)$ which collapses to the $2 k+2-$ valent vertex of $\Gamma_{p}$. Then every edge in $A(t)$ has length $\leq \frac{1}{2}$. If $\Gamma(t)$ has a massive point then it must be the center of mass of $A(t)$ so $A(t) \subseteq A_{j}(t)$ for any $j$ so that $t \in K_{j}$ (from the previous subsection). Consequently, Theorem 4.11] allows us to impose the radial framing on $A(t)$ centered at the center of mass $c(t)$.

We note that, by definition, the center of mass is either a vertex or the midpoint of the edge on which it lies. Consequently, when this edge collapses, both 
endpoints collapse to $c(t)$ simultaneously. Thus the worse case (23) does not occur since $|C(v)|=2$ implies $v=c\left(\Gamma_{0}\right)$ which implies that $\left|C\left(f_{1}(v)\right)\right| \geq$ 4. Therefore, the only contribution to the 0-cell cocycle $z^{k}$ comes from the associahedron $A_{2 k+3}$.

This proves:

Theorem 4.17 The 0-cell cocycle $z^{k}$ is non-zero only inside the Stasheff associahedron $A_{2 k+3}$ with a standard framing on its boundary. Consequently, the adjusted Miller-Morita-Mumford class $\widetilde{\kappa}_{k}$ is dual to the Witten cycle $\left[W_{k}\right]$ and thus proportional to $\left[c_{\mathcal{F} \text { at }}^{k}\right]$ in $H^{2 k}(\mathcal{F} a t ; \mathbb{Q})$.

\section{The calculation}

The adjusted Miller-Morita-Mumford class $\widetilde{\kappa}_{k}$ is given by the 0-cell cocycle $z^{k}$ on framed fat graphs which is zero outside of the Stasheff associahedron $A_{2 k+3}=\left|\mathcal{A}_{2 k+3}\right|$. In this section we construct an explicit framing for this associahedron and use it to calculate the proportionality constant between $\widetilde{\kappa}_{k}$, $\left[c_{\mathcal{F} a t}^{k}\right]$ and $\left[W_{k}\right]^{*}$. Namely:

\section{Theorem 5.1}

$$
\widetilde{\kappa}_{k}=-\frac{1}{2}\left[c_{\mathcal{F} a t}^{k}\right]=(-1)^{k+1} \frac{(k+1) !}{(2 k+2) !}\left[W_{k}\right]^{*}
$$

\subsection{Framing the associahedron $A_{2 k+3}$}

First we subdivide $A_{2 k+3}$ so that we have a collar neighborhood $\partial A_{2 k+3} \times I$ of its boundary $\partial A_{2 k+3}$ and a half-sized copy $A_{2 k+3}^{1 / 2}$ of the standard associahedron inside. On this half-sized copy we take the radial framing about the center of mass. Since $2 k+3$ is odd this center of mass will always be at a vertex. On the outside boundary we take the framing given in the last section.

The outside boundary $\partial A_{2 k+3}$ contains $2 k+3$ copies of the associahedron $A_{2 k+2}$. Following the procedure outlined above we are required to subdivide each of these associahedra to form a half-sized copy $A_{2 k+2}^{1 / 2}$. Then we take the radial framing centered at the center of mass of the tree which collapses to the $2 k+2$ valent vertex in the center of $A_{2 k+2}^{1 / 2}$. Figure 5 gives an accurate picture when $k=1$. 


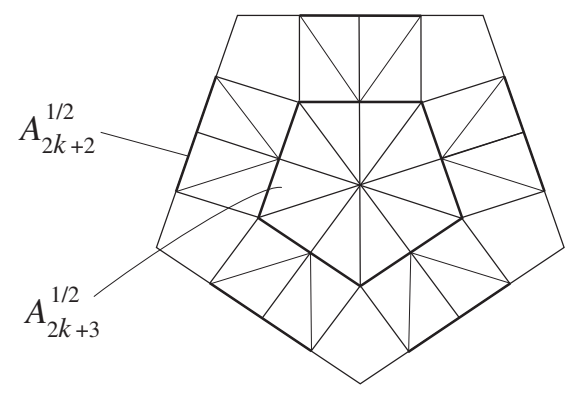

Figure 5: Subdivision of $A_{2 k+3}$

\subsection{Value of $z^{k}$ on $A_{2 k+3}^{1 / 2}$}

We first compute the value of the 0 -cell cocycle on the half-sized associahedron $A_{2 k+3}^{1 / 2}$. Since we are taking the radial framing only the center of mass is an unpaired 0-cell. Consequently, the only simplices in $A_{2 k+3}^{1 / 2}$ on which the 0 -cell cocycle $z^{k}$ has a chance of being non-zero are the ones in which at each step, $\Gamma_{i} \rightarrow \Gamma_{i+1}$, one more edge-vertex pair collapses into the center of mass.

Each time another vertex collapses to the center of mass another region is added to the middle. (Recall that a region is a gap between consecutive leaves.) There are $2 k+3$ regions. We start with three in the middle $a_{1}, a_{2}, a_{3}$ and we add regions $b_{1}, b_{2}, \cdots, b_{2 k}$ one at a time. The positions of these regions completely determine the shape of the tree in $\Gamma_{0}$ which collapses to the $2 k+3$ valent vertex of $\Gamma_{2 k}$ and the order of collapse determines (the collapsing part of) every graph $\Gamma_{i}$.

We will first count the number of $2 k$-simplices in $A_{2 k+3}^{1 / 2}$ obtained in this way. Then we will multiply by the average value of the 0 -cell cocycle on each term.

There are $2 k+1$ choices for $a_{1}$. Fixing $a_{1}=0$ we have:

$$
1 \leq a_{2} \leq k+1<a_{3} \leq a_{2}+k+1
$$

since $a_{1}, a_{2}, a_{3}$ are in cyclic order and there are no gaps greater than $k$ between two consecutive $a$ 's. (Otherwise the center of mass would be in that gap.) Consequently, there are $k+1$ choices for $a_{2}$ and the number of choices for $a_{3}$ is $a_{2}$ which has an average value of $\frac{k+2}{2}$. So there are

$$
\frac{1}{3}(2 k+3)(k+1)\left(\frac{k+2}{2}\right)
$$

choices for $\left(a_{1}, a_{2}, a_{3}\right)$. The factor $\frac{1}{3}$ comes from the fact that the numbering of the $a$ 's is only well-defined up to cyclic order. 
After the $a$ 's are chosen, the $b$ 's can be chosen arbitrarily. Thus there are $(2 k)$ ! choices for $b_{1}, \cdots, b_{2 k}$ making a total of

$$
\frac{1}{6}(2 k+3)(k+1)(k+2)(2 k) !
$$

terms. The orientation of the $2 k$-simplex is given by (Definition 3.11)

$$
\operatorname{sgn}\left(a_{1}, a_{2}, a_{3}, b_{1}, \cdots, b_{2 k}\right) .
$$

So the value of the 0 -cell cocycle on $A_{2 k+3}^{1 / 2}$ is

$$
\frac{1}{6}(2 k+3)(k+1)(k+2)(2 k) ! \frac{(-1)^{k} k ! E(Z)}{(2 k) !(2 k+3) ! / 2}=\frac{(-1)^{k}(k+2) ! E(Z)}{3(2 k+2) !}
$$

where $E(Z)$ is the expected value of

$$
Z=\sum_{i=1}^{3} \operatorname{sgn}\left(a_{i}, b_{1}, \cdots, b_{2 k}\right) \operatorname{sgn}\left(a_{1}, a_{2}, a_{3}, b_{1}, \cdots, b_{2 k}\right) .
$$

As in section 3 it is easier to compute

$$
Z+1=\left(1+(-1)^{P}\right)\left(1+(-1)^{Q}\right)
$$

where $P=a_{2}-a_{1}-1\left(=a_{2}-1\right.$ when $\left.a_{1}=0\right)$ and $Q=a_{3}-a_{2}-1$. The random variable $P$ takes values $0 \leq p \leq k$ with probability

$$
P(P=p)=\frac{p+1}{(k+1)(k+2) / 2} .
$$

The random variable $Q=a_{3}-a_{2}-1$ takes values $k-p \leq q \leq k$ with conditional probability

$$
P(Q=q \mid P=p)=\frac{1}{p+1}
$$

Thus

$$
P(Q \text { is even } \mid P=p)= \begin{cases}\frac{1}{2} & \text { for } p \text { odd } \\ \frac{1}{2}+\frac{(-1)^{k}}{2(p+1)} & \text { for } p \text { even. }\end{cases}
$$

So

$$
E(Z+1 \mid P=p)= \begin{cases}0 & \text { for } p \text { odd } \\ 2+\frac{(-1)^{k} 2}{p+1} & \text { for } p \text { even }\end{cases}
$$


and

$$
\begin{aligned}
E(Z+1) & =\sum_{j=0} E(Z+1 \mid P=2 j) P(P=2 j) \\
& =\sum_{j=0}^{\left[\frac{k}{2}\right]}\left(\frac{2\left(2 j+1+(-1)^{k}\right)}{2 j+1}\right)\left(\frac{2 j+1}{(k+1)(k+2) / 2}\right) \\
& =\sum_{j=0}^{\left[\frac{k}{2}\right]} \frac{4\left(2 j+1+(-1)^{k}\right)}{(k+1)(k+2)} .
\end{aligned}
$$

Case 1 If $k$ is odd then

$$
E(Z)=\frac{4}{(k+1)(k+2)}\left(\frac{k-1}{2}\right)\left(\frac{k+1}{2}\right)-1=\frac{-3}{k+2}
$$

so

$$
z^{k}\left(A_{2 k+3}^{1 / 2}\right)=\frac{(-1)^{k}(k+2) !}{3(2 k+2) !} \cdot \frac{-3}{k+2}=(-1)^{k+1} \frac{(k+1) !}{(2 k+2) !}
$$

Case 2 If $k$ is even then

so

$$
E(Z)=\frac{4}{(k+1)(k+2)}\left(\frac{k+2}{2}\right)\left(\frac{k+4}{2}\right)-1=\frac{3}{k+1}
$$

$$
z^{k}\left(A_{2 k+3}^{1 / 2}\right)=\frac{(-1)^{k}(k+2) !}{3(2 k+2) !} \cdot \frac{3}{k+1}=(-1)^{k} \frac{k !(k+2)}{(2 k+2) !} .
$$

\subsection{Value of $z^{k}$ on the collar}

The only $2 k$-simplices in the collar which have a chance of giving a non-zero value under $z^{k}$ are the ones in which $\Gamma_{0}$ contains a 0 -cell in the center of an edge $e_{0}$ which is the center of mass of an $A_{2 k+2}$ tree. The next graph $\Gamma_{1}$ must be in the boundary $\partial A_{2 k+3}^{1 / 2}$ where the center of mass shifts over to one of the endpoints $v_{1}$ of $\Gamma_{1}$ which must be equal to $\Gamma_{0}$.

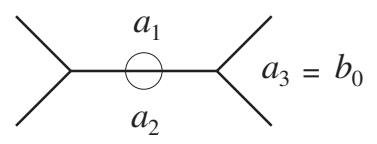

Figure 6: The center of mass (circle) shifts to $a_{3}=b_{0}$.

Using consistent notation with our previous discussion we label the first three angles $a_{1}, a_{2}, a_{3}$ as shown in Figure [6] where the circle represents the center of 
mass for $A_{2 k+2}^{1 / 2}$ and the right hand vertex is the center of mass for $A_{2 k+3}^{1 / 2}$. The next regions to converge to the center of mass are $b_{1}, \cdots, b_{2 k-1}$ with the region $b_{2 k}$ being left out. (See Figure 7 ) We note that the circled vertex should form a collapsing pair with the long edge $e$.

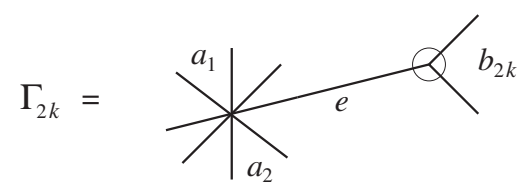

Figure 7: $b_{2 k}$ is left out.

In order for the center of mass of $A_{2 k+2}^{1 / 2}$ to be on the edge separating $a_{1}, a_{2}$ we must have $k$ regions on one side and $k+1$ regions on the other and $b_{2 k}$ must be in the part with $k+1$ regions. The region $a_{2}=b_{0}$ must also be on the same side as $b_{2 k}$ and our convention is that $a_{1}, a_{2}, a_{3}$ are in cyclic order. The other regions $b_{1}, \cdots, b_{2 k-1}$ can be places arbitrarily. The number of possible configurations is:

$$
(2 k+3)(k+1) k(2 k-1) !
$$

( $a_{1}$ can be chosen arbitrarily, there is no choice about $a_{2}$, there are $k+1$ choices for $b_{0}=a_{3}$ and only $k$ choices for $b_{2 k}$.)

The orientation of this $2 k$-simplex is given by

$$
-\operatorname{sgn}\left(a_{1}, a_{2}, b_{0}, \cdots, b_{2 k}\right)
$$

since $\Gamma_{0}$ is on the wrong side of the odd dimensional face $\Gamma_{1} \rightarrow \cdots \rightarrow \Gamma_{2 k-1}$. (See Figure 8)

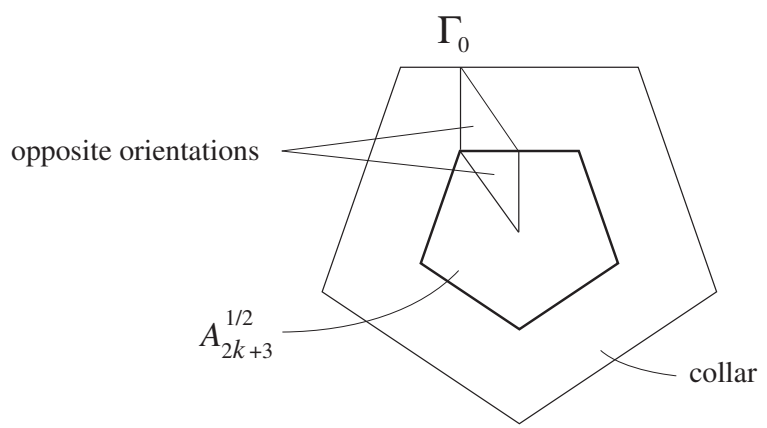

Figure 8: The orientation is reversed in the collar. 
The average value of the 0 -cell cocycle $z^{k}$ on the simplex is

$$
\frac{(-1)^{k} k !}{(2 k) !} \frac{E(W)}{(2 k+2) !}
$$

where $E(W)$ is the expected value of

$$
W=-\sum_{i=1}^{2} \operatorname{sgn}\left(a_{i}, b_{0}, \cdots, b_{2 k-1}\right) \operatorname{sgn}\left(a_{1}, a_{2}, b_{0}, \cdots, b_{2 k}\right) .
$$

If $k$ is odd then $W=0$ since the summands $\operatorname{sgn}\left(a_{i}, b_{0}, \cdots, b_{2 k-1}\right)$ have opposite sign.

If $k$ is even then

$$
E(W)=\frac{-2}{k+1}
$$

since the number of $b$ 's between $b_{2 k}$ and $a_{1}$ or $a_{2}$ is a random variable $N$ taking the values $0, \cdots, k$ with equal probability and $W=-2(-1)^{N}$.

Consequently, when $k$ is even, the total value of $z^{k}$ on the collar of $A_{2 k+3}$ is given by multiplying (28) and (29) using (30):

$$
-(2 k+3)(k+1) k(2 k-1) ! \frac{(-1)^{k} k !}{(2 k) !} \frac{-2}{k+1}=(-1)^{k+1} \frac{k !(2 k+3)}{(2 k+2) !} .
$$

Adding this to (27) gives:

$$
\frac{(-1)^{k} k !}{(2 k+2) !}[k+2-(2 k+3)]=(-1)^{k+1} \frac{(k+1) !}{(2 k+2) !}
$$

when $k$ is even.

When $k$ is odd we get the same answer (26) since $E(W)=0$. This completes the proof of Theorem 5.1 .

\section{References}

[1] E Arbarello, M Cornalba, Combinatorial and algebro-geometric cohomology classes on the moduli spaces of curves, J. Alg. Geom. 5 (1996) 705-749

[2] Marc Culler, Karen Vogtmann, Moduli of graphs and automorphisms of free groups, Invent. Math. 84 (1986) 91-119

[3] John L Harer, Stability of the homology of the mapping class groups of orientable surfaces, Ann. of Math. 21 (1985) 215-249

[4] Kiyoshi Igusa, Graph cohomology and Kontsevich cycles, to appear in Topology, arXiv:math.AT/0303157 
[5] Kiyoshi Igusa, The space of framed functions, Trans. Amer. Math. Soc. 301 (1987) 431-477

[6] Kiyoshi Igusa, A multiplication in cyclic homology, Trans. Amer. Math. Soc. 352 (2000) 209-242

[7] Kiyoshi Igusa, Higher Franz-Reidemeister Torsion, AMS/IP Studies in Advanced Mathematics 31, International Press (2002)

[8] Kiyoshi Igusa, Michael Kleber, Increasing trees and Kontsevich cycles, Geom. Topol. 8 (2004) 969-1012

[9] Kiyoshi Igusa, John Klein, The Borel regulator map on pictures. II. An example from Morse theory, $K$-Theory 7 (1993) 225-267

[10] Maxim Kontsevich, Intersection theory on the moduli space of curves and the matrix Airy function, Comm. Math. Phys. 147 (1992) 1-23

[11] Edward Y Miller, The homology of the mapping class group, J. Differential Geom. 24 (1986) 1-14

[12] Gabriele Mondello, Combinatorial classes on the moduli space of curves are tautological, to appear in IMRN, arXiv:math.AT/0303207

[13] Shigeyuki Morita, Characteristic classes of surface bundles, Bull. Amer. Math. Soc. 11 (1984) 386-388

[14] Shigeyuki Morita, Characteristic classes of surface bundles, Invent. Math. 90 (1987) 551-577

[15] Shigeyuki Morita, Stucture of the mapping class group: a survey and a prospect, from: "Proceedings of the Kirbyfest" (Joel Hass and Martin Scharlemann, editors), Geom. Topol. Monogr. 2 (1999) 349-406

[16] David Mumford, Towards an enumerative geometry of the moduli space of curves, from: "Arithmetic and geometry, Vol. II", Birkhäuser Boston, Boston, MA (1983) 271-328

[17] Robert C Penner, The decorated Teichmüller space of punctured surfaces, Comm. Math. Phys. 113 (1987) 299-339

[18] Robert C Penner, The Poincaré dual of the Weil-Petersson Kähler two-form, from: "Perspectives in mathematical physics", Internat. Press, Cambridge, MA (1994) 229-249

[19] J D Stasheff, Homotopy Associativity of H-spaces I,II, Trans. AMS 108 (1963) 275-292, 293-312

[20] Kurt Strebel, Quadratic Differentials, Springer-Verlag, Berlin (1984)

Department of Mathematics, Brandeis University

Waltham, MA 02454-9110, USA

Email: igusa@brandeis.edu

Received: 18 December 2003 Revised: 26 May 2004 